\title{
Ergodic Directions for Billiards in a Strip with Periodically Located Obstacles
}

\author{
Krzysztof Frączek $^{1}$, Corinna Ulcigrai ${ }^{2}$ \\ 1 Faculty of Mathematics and Computer Science, Nicolaus Copernicus University, ul. Chopina 12/18, \\ 87-100 Toruń, Poland. E-mail: fraczek@mat.umk.pl \\ 2 Department of Mathematics, University Walk, Clifton, Bristol BS8 1TW, UK. \\ E-mail: corinna.ulcigrai@bristol.ac.uk
}

Received: 30 August 2012 / Accepted: 20 December 2013

Published online: 26 March 2014 - (C) The Author(s) 2014. This article is published with open access at Springerlink.com

\begin{abstract}
We study the size of the set of ergodic directions for the directional billiard flows on the infinite band $\mathbb{R} \times[0, h]$ with periodically placed linear barriers of length $0<\lambda<h$. We prove that the set of ergodic directions is always uncountable. Moreover, if $\lambda / h \in(0,1)$ is rational, the Hausdorff dimension of the set of ergodic directions is greater than $1 / 2$. In both cases (rational and irrational) we construct explicitly some sets of ergodic directions.
\end{abstract}

\section{Introduction}

In this paper we consider the following infinite periodic billiard, whose ergodic properties have been the object of recent investigation (see e. g. [1,3,9]). Let $T(h, a, \lambda)$ be the billiard table (shown in Fig. 1) given by an infinite band $\mathbb{R} \times[0, h]$ with periodically placed linear barriers of length $0<\lambda<h$ handling from the lower side of the band perpendicularly, that is:

$$
T(h, a, \lambda)=(\mathbb{R} \times[0, h]) \backslash(a \mathbb{Z} \times[0, \lambda]) .
$$

A billiard trajectory is the trajectory of a point-mass which moves freely inside the table on segments of straight lines and undergoes elastic collisions (angle of incidence is equal to the angle of reflection) when it hits the boundary of the table. The billiard flow $\left(\varphi_{t}\right)_{t \in \mathbb{R}}$ is defined on the subset of the phase space $T(h, a, \lambda) \times S^{1}$ that consists of the points $(x, \theta) \in T(h, a, \lambda) \times S^{1}$ such that if $x$ belongs to the boundary of $T(h, a, \lambda)$ then $\theta$ is an inward direction. For $t \in \mathbb{R}$ and $(x, \theta)$ in the domain of $\left(\varphi_{t}\right)_{t \in \mathbb{R}}, \varphi_{t}$ maps $(x, \theta)$ to $\varphi_{t}(x, \theta)=\left(x_{t}, \theta_{t}\right)$, where $x_{t}$ is the point reached after time $t$ by flowing at unit speed along the billiard trajectory starting at $x$ in direction $\theta$ and $\theta_{t}$ is the tangent direction to the trajectory at $x_{t}$. A similar billiard in a semi-infinite band was studied in [1] in the context of perfect retroreflectors. For a survey on billiards in finite and infinite polygons we refer the reader to $[5,6]$. 


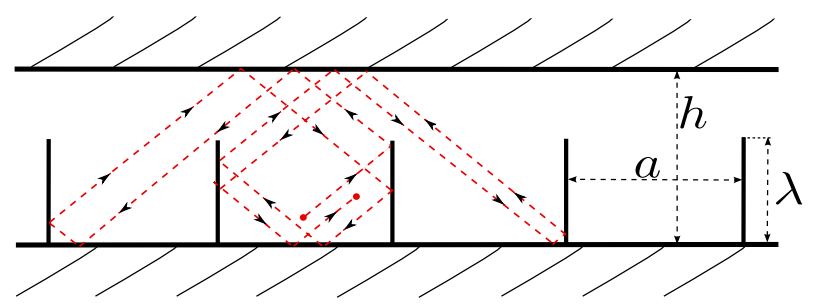

Fig. 1. Billiard flow on $T(h, a, \lambda)$

Denote by $\Gamma$ the 4-element group of isometries of $S^{1}$ generated by the reflections $\theta \mapsto \bar{\theta}, \theta \mapsto-\bar{\theta}$. For every direction $\theta \in S^{1}$ the billiard flow on $T(h, a, \lambda)$ has the invariant subset $T(h, a, \lambda) \times(\Gamma \theta)$ in the phase space. The billiard flow $\left(\varphi_{t}^{\theta}\right)_{t \in \mathbb{R}}$ restricted to this set preserves the product of the Lebesgue measure on $T(h, a, \lambda)$ and the counting measure on the orbit $\Gamma \theta$.

In [3] we proved the following result:

Theorem ([3]). If $\lambda / h$ is rational or belongs to a set $\Delta \subset(0,1)$ of full Lebesgue measure then for almost every direction $\theta$ the billiard flow $\left(\varphi_{t}^{\theta}\right)_{t \in \mathbb{R}}$ on $T(h, a, \lambda)$ is not ergodic.

It is hence natural to ask whether there are exceptional ergodic directions. Hubert and Weiss proved in [9] that if $\lambda / h$ is rational then the set of ergodic directions contains a dense $G_{\delta}$ set. The aim of this paper is to prove the existence of ergodic directions for all irrational values of the relative slit length $\lambda / h$. In addition, we also study the size of this exceptional set of ergodic directions in the rational case. More precisely, we prove that the set of ergodic directions is uncountable when $\lambda / h$ is irrational (see Theorem 4.1) and for rational $\lambda / h$ we prove that its Hausdorff dimension is greater than $1 / 2$ (see Theorem 5.1).

In both cases (rational and irrational) we give an explicit construction of ergodic directions by specifying their continued fraction expansions. The proofs use an ergodicity criterion from [9] (Theorem 2.1) based on an approximation of $\theta$ by directions with infinite strips. The main idea is to study the action of $S L(2, \mathbb{Z})$ on homology and to cleverly exploit the symmetries of the system to construct infinite strips.

Combining our results from Sect. 5 with the approach introduced recently by Hooper in [7] one might be able to describe all invariant ergodic Radon measures for $\left(\varphi_{t}^{\theta}\right)_{t \in \mathbb{R}}$ whenever $\lambda / h$ is rational and $\theta$ belongs to a set of positive Hausdorff dimension.

\section{Background Material}

2.1. Directional flows on translation surfaces and $\mathbb{Z}$-covers. The study of directional billiard flows on any rational polygon (not necessary compact) can be reduced via an unfolding procedure (see i.e. [10]) to the study of directional flows on a translation surface. The translation surface corresponding to the table $T(h, a, \lambda)$ will be described in Sect. 2.2. In this section we briefly recall some basic definitions related to the notion of translation surface.

Let $M$ be an oriented surface (not necessarily compact). A translation surface $(M, \omega)$ is a complex structure on $M$ together with an nonzero Abelian differential $\omega$, that is a non-zero holomorphic 1-form. Let $\Sigma=\Sigma_{\omega} \subset M$ be the set of zeros of $\omega$. For every 

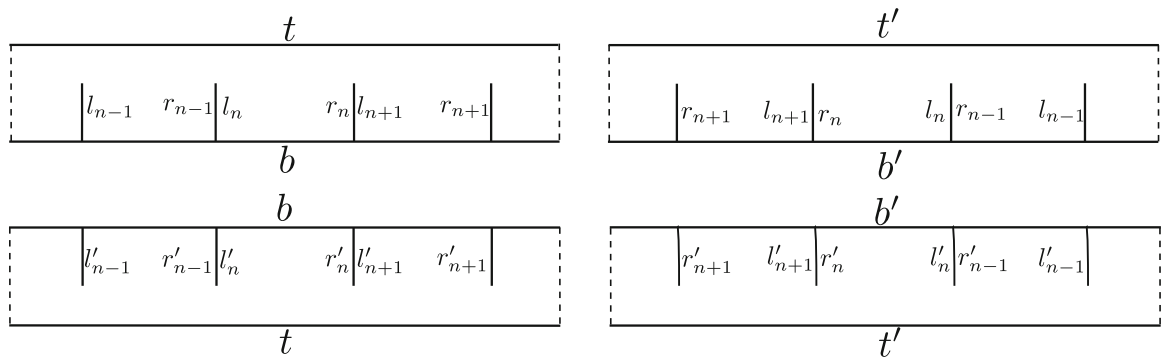

Fig. 2. Four copies of $T(h, a, \lambda)$

$\theta \in S^{1}=\mathbb{R} / 2 \pi \mathbb{Z}$ denote by $X_{\theta}=X_{\theta}^{\omega}$ the directional vector field in direction $\theta$ on $M \backslash \Sigma$, defined by $i_{X_{\theta}} \omega=e^{i \theta}$. Then the corresponding directional flow $\left(\phi_{t}^{\theta}\right)_{t \in \mathbb{R}}=\left(\phi_{t}^{\omega, \theta}\right)_{t \in \mathbb{R}}$ (also known as translation flow) on $M \backslash \Sigma$ preserves the area form $\nu_{\omega}=\frac{i}{2} \omega \wedge \bar{\omega}=$ $\Re(\omega) \wedge \Im(\omega)$. We will denote by $A(\omega):=v_{\omega}(M)$ the area of the surface.

Let $(M, \omega)$ be a compact connected translation surface. Denote by $\langle\cdot, \cdot\rangle: H_{1}(M, \mathbb{Z}) \times$ $H_{1}(M, \mathbb{Z}) \rightarrow \mathbb{Z}$ the algebraic intersection form.

Recall that a $\mathbb{Z}$-cover of $M$ is a surface $\widetilde{M}$ with a free totally discontinuous action of the group $\mathbb{Z}$ such that the quotient manifold $\widetilde{M} / \mathbb{Z}$ is homeomorphic to $M$. The map $p: \widetilde{M} \rightarrow$ $M$ obtained by composition of the projection $\widetilde{M} \rightarrow \widetilde{M} / \mathbb{Z}$ and the homeomorphism $\widetilde{M} / \mathbb{Z} \rightarrow M$ is called a covering map. Denote by $\widetilde{\omega}$ the pullback of the form $\omega$ by the map $p$. Then $(\widetilde{M}, \widetilde{\omega})$ is a translation surface as well. The translation flow on $(\widetilde{M}, \widetilde{\omega})$ in a direction $\theta$ will be denoted by $\left(\widetilde{\phi}_{t}^{\theta}\right)_{t \in \mathbb{R}}$.

All $\mathbb{Z}$-covers of $M$ (up to isomorphism) are in one-to-one correspondence with homology classes in $H_{1}(M, \mathbb{Z})$. The $\mathbb{Z}$-cover $\widetilde{M}_{\gamma}$ determined by $\gamma \in H_{1}(M, \mathbb{Z})$, under this correspondence, has the following properties. If $\sigma$ is a close curve in $M$ and $[\sigma] \in H_{1}(M, \mathbb{Z})$, then $\sigma$ lifts to a path $\widetilde{\sigma}:\left[t_{0}, t_{1}\right] \rightarrow \widetilde{M}_{\gamma}$ such that $\sigma\left(t_{1}\right)=n \cdot \sigma\left(t_{0}\right)$, where $n:=\langle\gamma,[\sigma]\rangle \in \mathbb{Z}$ and $\cdot$ denotes the action of $\mathbb{Z}$ on $\left(\widetilde{M}_{\gamma}, \widetilde{\omega}_{\gamma}\right)$ by deck transformations.

Denote by hol $: H_{1}(M, \mathbb{Z}) \rightarrow \mathbb{C}$ the holonomy map, i.e. $\operatorname{hol}(\gamma)=\int_{\gamma} \omega$ for every $\gamma \in H_{1}(M, \mathbb{Z})$. As shown by Hooper and Weiss (see Proposition 15 in [8]), $\operatorname{hol}(\gamma)=0$ if and only if for every $\theta \in S^{1}$ such that $\left(\phi_{t}^{\theta}\right)_{t \in \mathbb{R}}$ is ergodic, the flow $\left(\widetilde{\phi}_{t}^{\theta}\right)_{t \in \mathbb{R}}$ on the $\mathbb{Z}$-cover $\left(\widetilde{M}_{\gamma}, \widetilde{\omega}_{\gamma}\right)$ is recurrent. For this reason, following [8], if $\gamma \in H_{1}(M, \mathbb{Z})$ has $\operatorname{hol}(\gamma)=0$ we say that the $\mathbb{Z}$-cover $\left(\widetilde{M}_{\gamma}, \widetilde{\omega}_{\gamma}\right)$ of the translation surface $(M, \omega)$ given by $\gamma$ is recurrent.

2.2. From billiard flows to translation flows on translation surfaces. Fix parameters $(h, a, \lambda)$ and a direction $\theta \in S^{1}$. One can verify, using the unfolding process described for example in [10], that the flow $\left(\varphi_{t}^{\theta}\right)_{t \in \mathbb{R}}$ on the table $T(h, a, \lambda)$ is isomorphic to the directional flow $\left(\widetilde{\phi}_{t}^{\theta}\right)_{t \in \mathbb{R}}$ on a non-compact translation surface $(\widetilde{M}, \widetilde{\omega})$ which is obtained gluing four copies of $T(h, a, \lambda)$ along the segments of the same name, as shown in Fig. 2. Moreover, the surface $(\widetilde{M}, \widetilde{\omega})$ can be represented as gluing two $\mathbb{Z}$-periodic polygons (obtained by gluing pairs of copies of $T(h, a, \lambda)$ along $b$ and $\left.b^{\prime}\right)$ as shown in the Fig. 3 (here $R_{n}=r_{n} \cup r_{n}^{\prime}$ and $L_{n}=l_{n} \cup l_{n}^{\prime}$ ).

Next, let us cut these polygons into rectangles $P_{n}, P_{n}^{\prime}$ along the segments $U_{n}, U_{n}^{\prime}, n \in$ $\mathbb{Z}$ (see the Fig. 4). It follows that $(\widetilde{M}, \widetilde{\omega})$ is a $\mathbb{Z}$-cover of the compact translation surface $(M, \omega) \in \mathcal{M}(1,1)$ presented in the Fig. 4 . More precisely, $(\widetilde{M}, \widetilde{\omega})=\left(\widetilde{M}_{\gamma_{0}}, \widetilde{\omega}_{\gamma_{0}}\right)$, where 

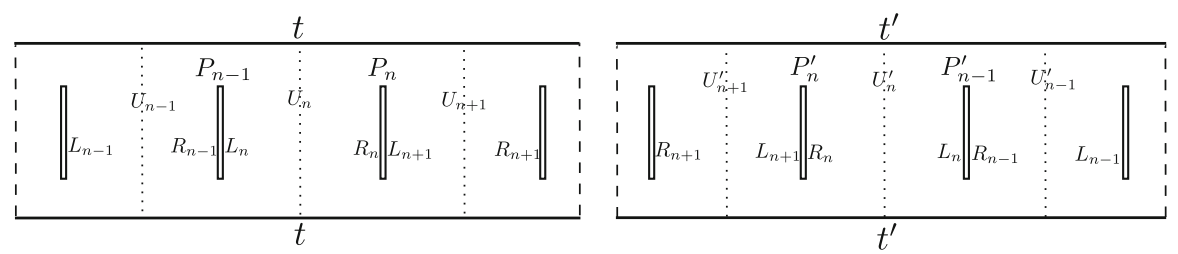

Fig. 3. The translation surface $(\widetilde{M}, \widetilde{\omega})$

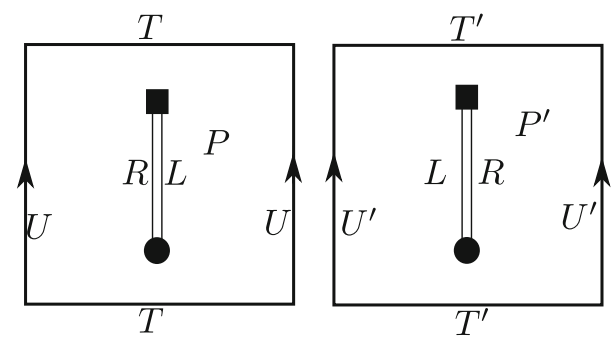

Fig. 4. The compact surface $(M, \omega)$

$\gamma_{0}=\left[U-U^{\prime}\right]$ and $\operatorname{hol}\left(\gamma_{0}\right)=0$. Consequently, the directional billiard flow $\left(\varphi_{t}^{\theta}\right)_{t \in \mathbb{R}}$ is isomorphic to the translation flow $\left(\widetilde{\phi}_{t}^{\theta}\right)_{t \in \mathbb{R}}$ on the recurrent $\mathbb{Z}$-cover $\left(\widetilde{M}_{\gamma_{0}}, \widetilde{\omega}_{\gamma_{0}}\right)$.

2.3. Moduli space and Teichmüller flow. Let $M$ be a compact connected oriented surface of genus $g$ and let $\Sigma \subset M$ be a finite set with cardinality $s \in \mathbb{N}$. Denote by $\operatorname{Diff}^{+}(M, \Sigma)$ the group of orientation-preserving homeomorphisms of $M$ preserving $\Sigma$. Denote by $\operatorname{Diff}_{0}^{+}(M, \Sigma)$ the subgroup of elements $\operatorname{Diff}^{+}(M, \Sigma)$ which are isotopic to the identity. Let us denote by $\Gamma(M, \Sigma):=\operatorname{Diff}^{+}(M, \Sigma) / \operatorname{Diff}_{0}^{+}(M, \Sigma)$ the mapping-class group.

Let $\kappa=\left(\kappa_{1}, \ldots, \kappa_{s}\right)$ be a family of natural numbers such that $2 g-2=\sum_{i=1}^{s} \kappa_{i}$. The stratum $\mathcal{M}(\kappa)=\mathcal{M}(M, \Sigma, \kappa)$ of the moduli space of Abelian differentials is the space of orbits of the natural action of $\operatorname{Diff}^{+}(M, \Sigma)$ on the space of all Abelian differentials on $M$ with $s$ zeros at $\Sigma$ of degrees $\kappa_{1}, \ldots, \kappa_{s}$. The stratum $\mathcal{Q}(M, \Sigma, \kappa)$ of the Teichmüller space of Abelian differentials is the space of orbits of the natural action of $\operatorname{Diff}_{0}^{+}(M, \Sigma)$ on the space of all Abelian differentials on $M$ with $s$ zeros at $\Sigma$ of degrees $\kappa_{1}, \ldots, \kappa_{s}$. Thus $\mathcal{M}(M, \Sigma, \kappa)=\mathcal{Q}(M, \Sigma, \kappa) / \Gamma(M, \Sigma)$.

The group $G L(2, \mathbb{R})$ acts naturally on $\mathcal{Q}(M, \Sigma, \kappa)$ and $\mathcal{M}(M, \Sigma, \kappa)$, by postcomposition with the charts defined by local primitives of the holomorphic 1-form. The Abelian differential obtained acting by $g \in G L(2, \mathbb{R})$ on $\omega$ will be denoted by $g \cdot \omega$. The Teichmüller flow $\left(G_{t}\right)_{t \in \mathbb{R}}$ is the restriction of this action to the diagonal subgroup $\left(\operatorname{diag}\left(e^{t}, e^{-t}\right)\right)_{t \in \mathbb{R}}$ of $G L(2, \mathbb{R})$ on $\mathcal{Q}(M, \Sigma, \kappa)$ and $\mathcal{M}(M, \Sigma, \kappa)$.

2.4. Ergodicity for $\mathbb{Z}$-periodic surfaces. In this section we formulate a result from [9] which provides an effective method to prove the ergodicity of translation flows on recurrent $\mathbb{Z}$-covers of compact translation surfaces and will be exploited to prove our main results.

Let $(M, \omega)$ be a compact connected translation surface. Let $\left(\widetilde{M}_{\gamma}, \widetilde{\omega}_{\gamma}\right)$ be one of its recurrent $\mathbb{Z}$-covers. Suppose that $C \subset M$ is a cylinder. Let $\delta(C) \in H_{1}(M, \mathbb{Z})$ be the homology class of any core curve of the cylinder $C$. We will use the following notation (introduced in [9]): 


$$
k(C):=\langle\delta(C), \gamma\rangle \in \mathbb{Z}, \quad v(C):=\operatorname{hol}(\delta(C)) \in \mathbb{R}^{2}, \quad A(C)>0 \text { is the area of } C .
$$

Note that if $k(C) \neq 0$ then the lift $\widetilde{C}_{\gamma} \subset \widetilde{M}_{\gamma}$ of $C$ to the $\mathbb{Z}$-cover $\widetilde{M}_{\gamma}$ is an infinite strip.

Definition 1 (see [9]). A direction $\theta \in S^{1}$ is well approximated by strips of the surface $\left(\widetilde{M}_{\gamma}, \widetilde{\omega}_{\gamma}\right)$ if there exist $\varepsilon, c>0, k_{\theta} \in \mathbb{Z} \backslash\{0\}$ and infinitely many strips $\widetilde{C} \subset \widetilde{M}_{\gamma}$ for which

$$
k(C)=k_{\theta}, \quad A(C)>c \quad \text { and } \quad|(\cos \theta, \sin \theta) \wedge v(C)| \leq(1-\varepsilon) \frac{A(C)}{2\|v(C)\|} .
$$

The following result follows directly from the proof of Theorem 1 in [9] (more precisely from Claim 12).

Theorem 2.1. Suppose that $\theta \in S^{1}$ is an ergodic direction for the translation flow on $(M, \omega)$. If $\theta \in S^{1}$ is well approximated by strips of the surface $\left(\widetilde{M}_{\gamma}, \widetilde{\omega}_{\gamma}\right)$ with $k_{\theta}= \pm 1$ then the flow $\left(\widetilde{\phi}_{t}^{\theta}\right)_{t \in \mathbb{R}}$ on $\left(\widetilde{M}_{\gamma}, \widetilde{\omega}_{\gamma}\right)$ is ergodic.

In order to prove ergodicity of the translation flow on $(M, \omega)$, which is required to apply the above Theorem, the following result from [11], know as Masur's criterion, will be helpful.

Theorem 2.2 (Masur's criterion [11]). Let $(M, \omega) \in \mathcal{M}(\kappa)$ be a compact translation surface. Let $g \in S L(2, \mathbb{R})$ be an element that maps the direction $\theta$ to the vertical direction. Suppose that there exists a bounded subset $B \subset \mathcal{M}(\kappa)$ and a sequence $t_{n} \rightarrow+\infty$ such that $G_{t_{n}}(g \cdot(M, \omega)) \in B$ for all $n \in \mathbb{N}$. Then the directional flow $\left(\phi_{t}^{\theta}\right)_{t \in \mathbb{R}}$ on $(M, \omega)$ is uniquely ergodic.

\section{Construction of Ergodic Directions}

In this section we describe a procedure to construct strips on the translation surface $\left(\widetilde{M}_{\gamma_{0}}, \widetilde{\omega}_{\gamma_{0}}\right.$ ) (see the end of Sect. 2.2) which will allow us to apply Theorem 2.1. We will study the behavior of the $S L(2, \mathbb{Z})$-orbit of $\gamma_{0} \in H_{1}(M, \mathbb{Z})$ for the $S L(2, \mathbb{Z})$ action induced on $H_{1}(M, \mathbb{Z})$. For simplicity, we always assume that $a=1=2 h$, then $\lambda=|L| / 2=|R| / 2 \in(0,1 / 2)$.

Let $\mathbb{T}_{0}^{2}$ denote the set

$$
\mathbb{T}_{0}^{2}:=[-1 / 2,1 / 2) \times[-1 / 2,1 / 2) \backslash\{(0,0),(-1 / 2,-1 / 2),(-1 / 2,0),(0,-1 / 2)\} .
$$

For $z \in \mathbb{T}_{0}^{2}$, let $M(z) \in \mathcal{M}(1,1)$ be the translation surface drawn in Fig. 5. We will distinguish between the singular points ( $\bullet$ and $\boldsymbol{\square}$ in Fig. 5) and $z=(x, y)$ will denote the position of the singular point $\mathbf{\square}$ wile $-z$ will be the position of the singular point - Let $\mathscr{L} \subset \mathcal{M}(1,1)$ be the locus containing all the surfaces $M(z), z \in \mathbb{T}_{0}^{2}$. Notice that the group $\operatorname{Aut}(M(z))$ of automorphisms of the translation surface fixing singular points consists of the identity map $i d$ and of the map $\tau: M(z) \rightarrow M(z)$ which exchanges the two squares (tori) in $M(z)$ by translations.

For every $M(z) \in \mathscr{L}$ let us fix the basis (called a standard basis) $\{\alpha, \beta\}$ of the subgroup

$$
H_{1}^{(0)}(M(z), \mathbb{Z})=\left\{\gamma \in H_{1}(M(z), \mathbb{Z}): \tau_{*} \gamma=-\gamma\right\}
$$

as in Fig. 5. Then $(M, \omega)=M(0, \lambda) \in \mathscr{L}$ and $\gamma_{0}=\beta$. 

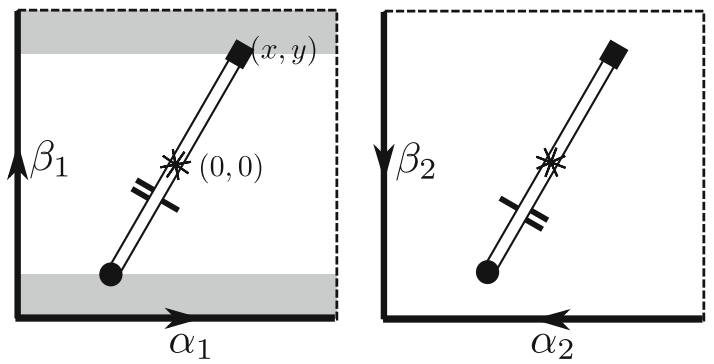

$$
\begin{gathered}
\alpha:=\alpha_{1}+\alpha_{2} \\
\beta:=\beta_{1}+\beta_{2}
\end{gathered}
$$

Fig. 5. The translation surface $M(x, y)$ and the standard basis $\{\alpha, \beta\}$ of $H_{1}^{(0)}(M(x, y), \mathbb{Z})$

The group $G L(2, \mathbb{R})$ acts naturally on $\mathbb{R}^{2}$ by matrix multiplication. We will denote the image of $z=(x, y) \in \mathbb{R}^{2}$ under $g \in G L(2, \mathbb{R})$ by $g(x, y)$ or $g z$. This action induces also a natural action $G L(2, \mathbb{Z}) \subset G L(2, \mathbb{R})$ on the torus $\mathbb{T}_{0}^{2}$. We will also denote by $g z \in \mathbb{T}_{0}^{2}$ the image of $z \in \mathbb{T}_{0}^{2}$ by the automorphism of $\mathbb{T}_{0}^{2}$ induced by $g$. One can show that for every $g \in G L(2, \mathbb{Z})$ :

$$
g \cdot M(z) \text { is identified with } M(g z) \text { in } \mathcal{M}(1,1)
$$

Thus the locus $\mathscr{L}$ is $G L(2, \mathbb{Z})$-invariant. In order to verify (2), it suffices to check it on generators of $G L(2, \mathbb{Z})$, for example on two matrices

$$
h^{+}:=\left(\begin{array}{ll}
1 & 1 \\
0 & 1
\end{array}\right), \quad \vartheta:=\left(\begin{array}{ll}
0 & 1 \\
1 & 0
\end{array}\right) .
$$

The matrix $\vartheta$ reflects the squares of $M(z)$ across their diagonal and the resulting surface has the form $M(\vartheta z)$. The equality $h^{+} \cdot M(z)=M\left(h^{+} z\right)$ is obtained as a byproduct of the proof of Lemma 3.3.

Since $\operatorname{Aut}(M(z))=\{i d, \tau\}$, there exist exactly two affine maps $\zeta^{g}, \tau \circ \zeta^{g}: M(z) \rightarrow$ $g \cdot M(z)$ whose derivatives are equal to $g$. Thus, if we denote by $\zeta_{*}: H_{1}^{(0)}(M(z), \mathbb{Z}) \rightarrow$ $H_{1}^{(0)}\left(M\left(z^{\prime}\right), \mathbb{Z}\right)$ the action on homology induced by a diffeomorphism $\zeta: M(z) \rightarrow$ $M\left(z^{\prime}\right)$, we have $\left(\tau \circ \zeta^{g}\right)_{*}=-\zeta_{*}^{g}$. Therefore, the induced homology action $g_{*}(z)$ : $H_{1}^{(0)}(M(z), \mathbb{Z}) \rightarrow H_{1}^{(0)}(g \cdot M(z), \mathbb{Z})$ is well defined up to \pm , i.e. $g_{*}(z)= \pm \zeta_{*}^{g}$. Representing this action in standard basis we obtain a matrix, denoted by $g_{*}(z)$, which is an element of $P G L(2, \mathbb{Z})$. Finally note that for all $g_{1}, g_{2} \in G L(2, \mathbb{Z})$ we have

$$
\left(g_{1} \cdot g_{2}\right)_{*}(z)=\left(g_{1}\right)_{*}\left(g_{2} z\right) \cdot\left(g_{2}\right)_{*}(z) .
$$

3.1. Construction of strips and an ergodicity criterion. We now present the procedure of construction of infinite family of infinite strips required by Theorem 2.1.

For any $z=(x, y) \in \mathbb{T}_{0}^{2}$ let us consider the cylinder

$$
C_{z}=[-1 / 2,1 / 2) \times([y, 1 / 2) \cup[-1 / 2,-y]) \quad \text { in } \quad M(z),
$$

which is shaded in Fig. 5. The holonomy vector of the core $\delta\left(C_{z}\right)$ of $C_{z}$ is $v\left(C_{z}\right)=(1,0)$ and $A\left(C_{z}\right)=1-2 y$, moreover $\left\langle\delta\left(C_{z}\right), \beta\right\rangle=1$. 
For any $g \in S L(2, \mathbb{Z})$ let $z_{g}=\left(x_{g}, y_{g}\right):=g^{-1}(z) \in \mathbb{T}_{0}^{2}$ and $\zeta^{g}: M\left(z_{g}\right) \rightarrow M(z)$ be an affine transformation whose derivative is $g \in S L(2, \mathbb{Z})$. Then $C_{g}:=\zeta^{g}\left(C_{z_{g}}\right) \subset M(z)$ is a cylinder such that $\delta\left(C_{g}\right)=\zeta_{*}^{g}\left(\delta\left(C_{z_{g}}\right)\right) \in H_{1}(M, \mathbb{Z})$ and

$$
\begin{gathered}
v\left(C_{g}\right)=\operatorname{hol}\left(\zeta_{*}^{g}\left(\delta\left(C_{z_{g}}\right)\right)\right)=D \zeta^{g} \operatorname{hol}\left(\delta\left(C_{z_{g}}\right)\right)=g(1,0), \\
k\left(C_{g}\right)=\left\langle\zeta_{*}^{g}\left(\delta\left(C_{z_{g}}\right)\right), \beta\right\rangle=\left\langle\delta\left(C_{z_{g}}\right),\left(\zeta_{*}^{g}\right)^{-1} \beta\right\rangle \\
A\left(C_{g}\right)=A\left(\zeta^{g}\left(C_{z_{g}}\right)\right)=A\left(C_{z_{g}}\right)=1-2 y_{g}
\end{gathered}
$$

Suppose additionally that $g_{*}\left(z_{g}\right) \beta=\beta$. Then $\zeta_{*}^{g} \beta= \pm \beta$, hence

$$
k\left(C_{g}\right)=\left\langle\delta\left(C_{z_{g}}\right),\left(\zeta_{*}^{g}\right)^{-1} \beta\right\rangle= \pm\left\langle\delta\left(C_{z_{g}}\right), \beta\right\rangle= \pm 1 .
$$

In summary, the above procedure allows to construct strips on $\widetilde{M(z)}{ }_{\beta}$ satisfying (1) whenever

$$
g_{*}\left(g^{-1}(z)\right) \beta=\beta .
$$

Combining this with Theorem 2.2 (Masur's criterion) and Theorem 2.1 (Hubert-Weiss criterion) yields the following criterion for the ergodicity of directional flows on the surface $\widehat{M(z)}{ }_{\beta}$.

Denote by $h^{-}$the transpose of the matrix $h^{+}$.

Theorem 3.1. Suppose that $z_{0} \in \mathbb{T}_{0}^{2}, \alpha=\left[0 ; a_{1}, a_{2}, a_{3}, \ldots\right], 0<a<b<1 / 2, \varepsilon>0$ and there exists an increasing sequence of even numbers $\left(k_{n}\right)_{n \geq 1}$ such that

$$
\left(\left(h^{+}\right)^{a_{1}} \cdots\left(h^{-}\right)^{a_{k_{n}}}\right)^{-1} M\left(z_{0}\right)=M\left(z_{n}\right),\left(\left(h^{+}\right)^{a_{1}} \cdots\left(h^{-}\right)^{a_{k_{n}}}\right)_{*}^{-1}\left(z_{0}\right) \beta=\beta,
$$

and setting $\left(x_{n}, y_{n}\right)=z_{n}$ we have

$$
a \leq y_{n} \leq b \quad \text { and } \quad a_{k_{n}+1} \geq \frac{1+\varepsilon}{1 / 2-y_{n}} .
$$

Then the directional flow in direction $(1, \alpha)$ on the $\mathbb{Z}$-cover $\widetilde{M\left(z_{0}\right)}$ $\beta$ given by $\beta$ is ergodic.

Proof. First we will show that the directional flow in direction $(1, \alpha)$ on the surface $M\left(z_{0}\right)$ is ergodic. Let $\left(p_{n} / q_{n}\right)_{n \geq 0}$ stand for the sequence of convergents of the continued fraction of $\alpha$. Then

Setting

$$
\left(h^{+}\right)^{a_{1}} \cdots\left(h^{-}\right)^{a_{k_{n}}}=\left(\begin{array}{ll}
q_{k_{n}} & q_{k_{n}-1} \\
p_{k_{n}} & p_{k_{n}-1}
\end{array}\right) .
$$

$$
\sigma:=\left(\begin{array}{cc}
\alpha & -1 \\
0 & 1 / \alpha
\end{array}\right)
$$

it is clear that $\sigma$ maps the direction of the vector $(1, \alpha)$ to the vertical direction. We will prove that the sequence

$$
\left(G_{\log q_{k_{n}}} \cdot \sigma \cdot M\left(z_{0}\right)\right)_{n \geq 1}
$$

is bounded in the moduli space. Indeed, by assumption,

$$
G_{\log q_{k_{n}}} \cdot \sigma \cdot M\left(z_{0}\right)=\sigma_{n} \cdot M\left(z_{n}\right),
$$


where

$$
\begin{aligned}
\sigma_{n} & :=\operatorname{diag}\left(q_{k_{n}}, 1 / q_{k_{n}}\right) \cdot \sigma \cdot\left(h^{+}\right)^{a_{1}} \cdots\left(h^{-}\right)^{a_{k_{n}}} \\
& =\left(\begin{array}{cc}
q_{k_{n}} & 0 \\
0 & 1 / q_{k_{n}}
\end{array}\right)\left(\begin{array}{cc}
\alpha & -1 \\
0 & 1 / \alpha
\end{array}\right)\left(\begin{array}{cc}
q_{k_{n}} & q_{k_{n}-1} \\
p_{k_{n}} & p_{k_{n}-1}
\end{array}\right) \\
& =\left(\begin{array}{cc}
q_{k_{n}}\left(q_{k_{n}} \alpha-p_{k_{n}}\right) & q_{k_{n}}\left(q_{k_{n}-1} \alpha-p_{k_{n}-1}\right) \\
p_{k_{n}} /\left(\alpha q_{k_{n}}\right) & p_{k_{n}-1} /\left(\alpha q_{k_{n}}\right)
\end{array}\right) .
\end{aligned}
$$

Moreover,

$$
\left|q_{k_{n}}\left(q_{k_{n}} \alpha-p_{k_{n}}\right)\right|<\left|q_{k_{n}}\left(q_{k_{n}-1} \alpha-p_{k_{n}-1}\right)\right|<1
$$

and, since $k_{n}$ is by construction even, so that $p_{k_{n}} / q_{k_{n}}<\alpha$, we have

$$
0<\frac{p_{k_{n}-1}}{\alpha q_{k_{n}}}<\frac{p_{k_{n}}}{\alpha q_{k_{n}}}<1 .
$$

Therefore, all $\sigma_{n}$ belong to the subset $G_{0} \subset S L(2, \mathbb{R})$ of matrices whose coefficients belong to $[-1,1]$. Of course, the set $G_{0}$ is compact. Let us consider the set

$$
B_{0}:=\{M(x, y) \in \mathscr{L}: y \in[a, b]\},
$$

which is compact in the moduli space $\mathcal{M}(1,1)$. Thus $G_{0} \cdot B_{0} \subset \mathcal{M}(1,1)$ is a compact subset in the moduli space $\mathcal{M}(1,1)$ as well. Since $\sigma_{n} \cdot M\left(z_{n}\right) \in G_{0} \cdot B_{0}$ for every natural $n$, in view of Theorem 2.2, the directional flow in direction $(1, \alpha)$ on the surface $M\left(z_{0}\right)$ is ergodic.

In the rest of the proof, Theorem 2.1 combined with the construction described before Theorem 3.1 will give the ergodicity of directional flow in direction $(1, \alpha)$ on the $\mathbb{Z}$-cover $\widehat{M\left(z_{0}\right)}{ }_{\beta}$.

Recall that for any $z=(x, y) \in \mathbb{T}_{0}^{2}$ we denote by $C_{z}$ the cylinder $[-1 / 2,1 / 2) \times$ $([y, 1 / 2) \cup[-1 / 2,-y]) \subset M(z)$. The holonomy vector of the core $\delta\left(C_{z}\right)$ of $C_{z}$ is $v\left(C_{z}\right)=(1,0)$, Area $\left(C_{z}\right)=1-2 y$ and $\left\langle\delta\left(C_{z}\right), \beta\right\rangle=1$.

By assumption, for every $n \geq 1$ there exists an affine transformation

$$
\zeta_{n}: M\left(z_{n}\right) \rightarrow M\left(z_{0}\right)
$$

whose derivative $D \zeta_{n}$ is $\left(h^{+}\right)^{a_{1}} \ldots\left(h^{-}\right)^{a_{k_{n}}}=\left(\begin{array}{cc}q_{k_{n}} & q_{k_{n}-1} \\ p_{k_{n}} & p_{k_{n}-1}\end{array}\right)$ and $\left(\zeta_{n}\right)_{*} \beta=\beta$. Let us consider the cylinder $C_{n}:=\zeta_{n}\left(C_{z_{n}}\right) \subset M\left(z_{0}\right)$ for which the homology class of the core is $\delta\left(C_{n}\right)=\left(\zeta_{n}\right)_{*}\left(\delta\left(C_{z_{n}}\right)\right)$. Then

$$
\begin{gathered}
k\left(C_{n}\right)=\left\langle\delta\left(C_{n}\right), \beta\right\rangle=\left\langle\left(\zeta_{n}\right)_{*}\left(\delta\left(C_{z_{n}}\right)\right),\left(\zeta_{n}\right)_{*}(\beta)\right\rangle=\left\langle\delta\left(C_{z_{n}}\right), \beta\right\rangle=1, \\
v\left(C_{n}\right)=\left(D \zeta_{n}\right) v\left(C_{z_{n}}\right)=\left(D \zeta_{n}\right)(1,0)=\left(q_{k_{n}}, p_{k_{n}}\right), \\
A\left(C_{n}\right)=A\left(C_{z_{n}}\right)=1-2 y_{n} .
\end{gathered}
$$

Therefore each cylinder $C_{n} \subset M\left(z_{0}\right)$ is lifted to an infinite strip $\widetilde{C}_{n} \subset \widetilde{M\left(z_{0}\right)_{\beta}}$. Using this sequence of strips we can show that the direction $(1, \alpha)$ is well approximated by strips. Indeed,

$$
\frac{\left|(1, \alpha) \wedge v\left(C_{n}\right)\right|}{\|(1, \alpha)\|}=\frac{\left|(1, \alpha) \wedge\left(q_{k_{n}}, p_{k_{n}}\right)\right|}{\|(1, \alpha)\|}=\frac{\left|q_{k_{n}} \alpha-p_{k_{n}}\right|}{\|(1, \alpha)\|}<\frac{1}{\|(1, \alpha)\|} \frac{1}{a_{k_{n}+1} q_{k_{n}}}
$$


and

$$
\frac{A\left(C_{n}\right)}{2\left\|v\left(C_{n}\right)\right\|} \geq \frac{1 / 2-y_{n}}{q_{k_{n}}\|(1, \alpha)\|}
$$

Therefore,

$$
\frac{\left|(1, \alpha) \wedge v\left(C_{n}\right)\right|}{\|(1, \alpha)\|} \leq \frac{1}{1+\varepsilon} \frac{A\left(C_{n}\right)}{2\left\|v\left(C_{n}\right)\right\|}
$$

whenever $a_{k_{n}+1} \geq(1+\varepsilon) /\left(1 / 2-y_{n}\right)$.

The ergodicity of the directional flow in direction $(1, \alpha)$ on the surface $\widetilde{M\left(z_{0}\right)}{ }_{\beta}$ hence follows directly from Theorem 2.1.

3.2. $S L_{+}(2, \mathbb{Z})$-action induced on homologies. Denote by $S L_{+}(2, \mathbb{Z})$ the semi-group of non-negative matrices in $S L(2, \mathbb{R})$. In this section we establishes some principal rules of the $S L_{+}(2, \mathbb{Z})$-action induced on homologies. These rules will help us to find elements $g \in S L_{+}(2, \mathbb{Z})$ satisfying (4), first for irrational $\lambda$ in Sect. 4 and then for rational $\lambda$ in Sect. 5.

Set

$$
h^{+}:=\left(\begin{array}{ll}
1 & 1 \\
0 & 1
\end{array}\right), \quad h^{-}:=\left(\begin{array}{ll}
1 & 0 \\
1 & 1
\end{array}\right), \quad \omega:=\left(\begin{array}{cc}
0 & 1 \\
-1 & 0
\end{array}\right), \quad \vartheta:=\left(\begin{array}{ll}
0 & 1 \\
1 & 0
\end{array}\right) .
$$

Then $S L_{+}(2, \mathbb{Z})$ is generated by $h^{+}$and $h^{-}$and one can check that:

$$
\begin{aligned}
& \vartheta \cdot h^{ \pm} \cdot \vartheta^{-1}=h^{\mp}, \quad \vartheta \cdot \omega^{ \pm 1} \cdot \vartheta^{-1}=\omega^{\mp 1}, \quad \omega \cdot h^{ \pm} \cdot \omega^{-1}=\left(h^{\mp}\right)^{-1}, \\
& h^{-} \cdot\left(h^{+}\right)^{-1} \cdot h^{-}=\omega^{-1}, \quad h^{+} \cdot\left(h^{-}\right)^{-1} \cdot h^{+}=\omega .
\end{aligned}
$$

Let us consider two involutions $-i d$ and $\vartheta$ in $G L(2, \mathbb{Z})$. They generate two involutions (denoted also by $-i d$ and $\vartheta$ ) acting on the locus $\mathscr{L}$. Geometrically,

- $-i d$ rotates the squares of $M(z)$ by angle $\pi$, therefore its induced action on $H_{1}^{(0)}(M, \mathbb{Z})$ maps $\alpha$ to $-\alpha$ and $\beta$ to $-\beta$ except when $z$ belongs to the boundary of $\mathbb{T}_{0}^{2}$;

- $\vartheta$ reflects the squares of $M(z)$ across their diagonal, therefore its action on $H_{1}^{(0)}(M, \mathbb{Z})$ exchanges the basis elements $\alpha$ and $\beta$.

These two involutions (symmetries) will help us to describe the action of $S L_{+}(2, \mathbb{Z})$ on the homology level. For this purpose, we will use the following two lemmas.

Lemma 3.2. Set $E:=\left\{(x, y) \in \mathbb{T}_{0}^{2}: x, y>-1 / 2\right\}$. For every $z \in \mathbb{T}_{0}^{2}$ we have

$$
\vartheta_{*}(z)=\vartheta \quad \text { and } \quad(-i d)_{*}(z)=i d \text { whenever } z \in E .
$$

Suppose that $g \cdot M(z)=M\left(z^{\prime}\right)$ for some $g \in S L(2, \mathbb{Z})$. Then

$$
\left(\vartheta \cdot g \cdot \vartheta^{-1}\right) \cdot M(\vartheta z)=M\left(\vartheta z^{\prime}\right) \text { and }\left(\vartheta \cdot g \cdot \vartheta^{-1}\right)_{*}(\vartheta z)=\vartheta \cdot g_{*}(z) \cdot \vartheta^{-1} \text {. }
$$

If additionally $z, z^{\prime} \in E$ then

$$
g \cdot M(-z)=M\left(-z^{\prime}\right) \text { and } g_{*}(-z)=g_{*}(z) .
$$


(a)

(d)
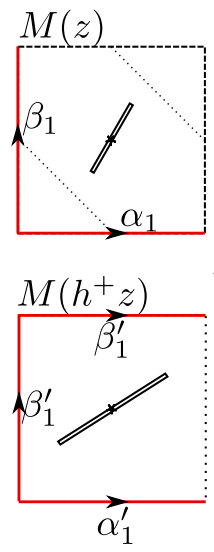

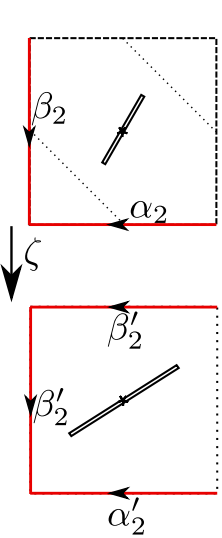

(b)

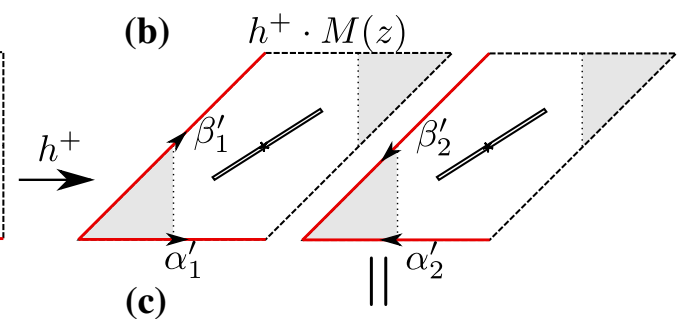

(c)
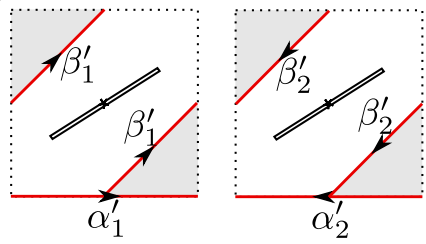

Fig. 6. The action of $h^{+}$on $M(z)$ if $z \in S$

Proof. The first claim (7) follows from the observations formulated before the lemma. The equality $\left(\vartheta \cdot g \cdot \vartheta^{-1}\right) \cdot M(\vartheta z)=M\left(\vartheta z^{\prime}\right)$ follows directly from the fact that $z^{\prime}=g z$ and (2). In view of (3),

$$
\left(\vartheta \cdot g \cdot \vartheta^{-1}\right)_{*}(\vartheta z)=\vartheta_{*}(g z) \cdot g_{*}(z) \cdot \vartheta_{*}^{-1}(\vartheta z) .
$$

Since $\vartheta^{-1}=\vartheta$, from (7) we obtain (8).

The equality $g \cdot M((-i d) z)=M\left((-i d) z^{\prime}\right)$ also follows directly from (2) and the fact that $-i d$ and $g$ commute. Moreover, in view of (3) and (7) applied to $z, z^{\prime} \in E$,

$$
\begin{aligned}
g_{*}(z) & =(-i d)_{*}\left(z^{\prime}\right) \cdot g_{*}(z)=((-i d) \cdot g)_{*}(z)=(g \cdot(-i d))_{*}(z) \\
& =g_{*}(-z) \cdot(-i d)_{*}(z)=g_{*}(-z) .
\end{aligned}
$$

Lemma 3.3. Set $S:=\left\{(x, y) \in \mathbb{T}_{0}^{2}:-1 / 2 \leq x+y<1 / 2\right\}$. For every $z \in \mathbb{T}_{0}^{2}$ we have

$$
h_{*}^{ \pm}(z)=\left\{\begin{array}{cc}
h^{ \pm} & \text {if } z \in S \\
\left(h^{ \pm}\right)^{-1} & \text { if } z \notin S
\end{array}\right.
$$

Proof. In Figs. 6 and 7 we present the surface $h^{+} \cdot M(z)$ for $z \in S$ and $z \notin S$ respectively and using cutting and pasting we show how to represent $h^{+} \cdot M(z)$ as $M\left(h^{+} z\right)$. The cut and paste in Fig. 6 is straightforward. After the linear action of $h^{+}$we cut and paste the shaded areas in order to represent the resulting surface as the union of two squares. Then the translation surface that we obtain is $M\left(h^{+} z\right)$.

Let us explain Fig. 7: after the linear action of $h^{+}$and a first cut and paste (as in the previous case), we consider the shaded areas labeled by $A, A^{\prime}, B$ and $B^{\prime}$ in Fig. 7. Recalling the gluings between slits, one can verify that $A$ and $A^{\prime}$ can be exchanged and similarly $B$ and $B^{\prime}$. The surface that we obtain after this operation is one of the canonical representatives of the locus $\mathscr{L}$, more precisely it is $M\left(z^{\prime}\right)$, where $z^{\prime}=h^{+} z$.

Let us denote by $\zeta=\zeta^{h^{+}}: M(z) \rightarrow M\left(h^{+} z\right)$ the affine map obtained combining the linear action of $h^{+}$with the cut and paste operations. By construction, we have $D \zeta=h^{+}$. In order to describe its induced action $\zeta_{*}$ on homology, in Figs. 6 and 7 we draw the 
(a)

(g)

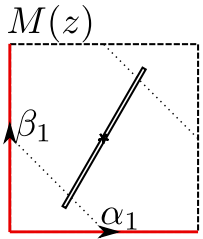

(h)
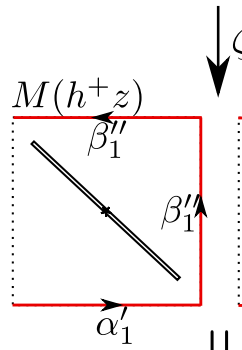

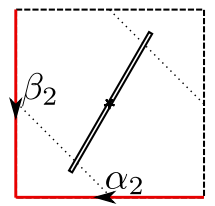

$\downarrow \zeta$

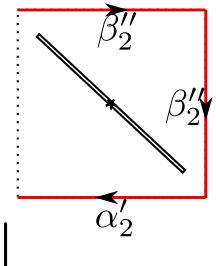

(f)
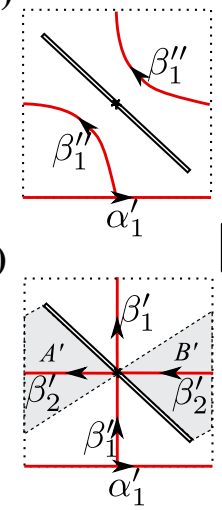

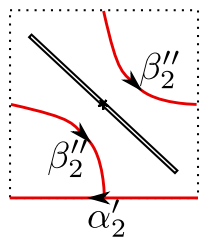

$\|$

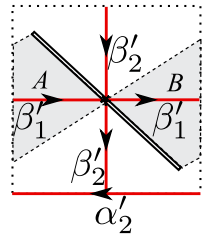

(b)

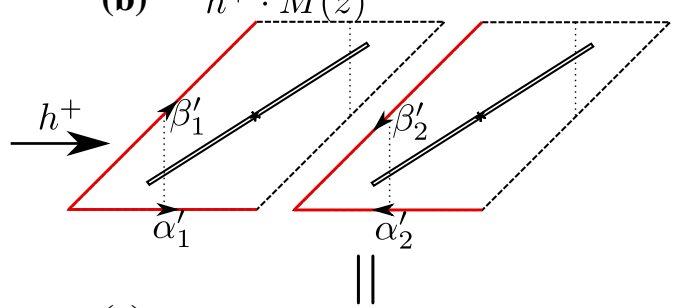

(c)

(d)
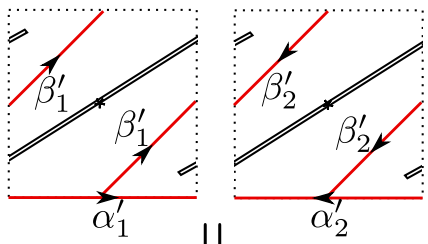

(e)
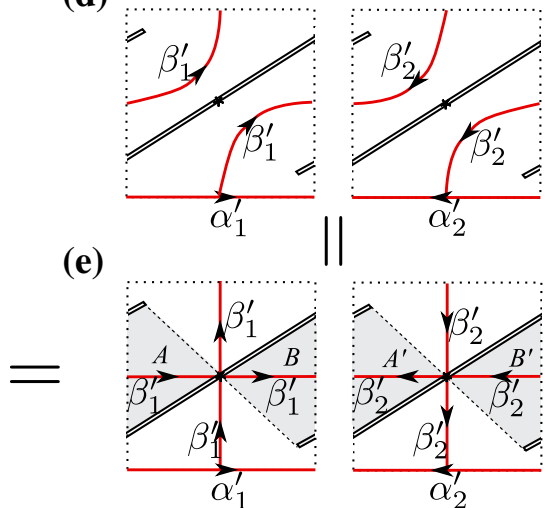

Fig. 7. The action of $h^{+}$on $M(z)$ if $z \notin S$

images of the homology classes of $\alpha, \beta$ under $\zeta_{*}$. Let us represent $\alpha=\alpha_{1}+\alpha_{2}$ and $\beta=\beta_{1}+\beta_{2}$ as the sum of two loops. Next set $\alpha_{1}^{\prime}=\zeta_{*}\left(\alpha_{1}\right), \alpha_{2}^{\prime}=\zeta_{*}\left(\alpha_{2}\right), \beta_{1}^{\prime}=\zeta_{*}\left(\beta_{1}\right)$ and $\beta_{2}^{\prime}=\zeta_{*}\left(\beta_{2}\right)$.

If $z \in S$ then, by changing representatives as shown in Fig. 6, one can verify that:

$$
\alpha_{1}^{\prime}=\alpha_{1}, \quad \alpha_{2}^{\prime}=\alpha_{2}, \quad \beta_{1}^{\prime}=\alpha_{1}+\beta_{1}, \quad \beta_{2}^{\prime}=\alpha_{2}+\beta_{2},
$$

so $\zeta_{*}(\alpha)=\alpha, \zeta_{*}(\beta)=\alpha+\beta$. Therefore, $\left(h^{+}\right)_{*}(z)=h^{+}$if $z \in S$.

If $z \notin S$ then, by changing representatives as shown in Fig. 7, one can verify that $\alpha_{1}^{\prime}=\alpha_{1}$ and $\alpha_{2}^{\prime}=\alpha_{2}$, so $\zeta_{*}(\alpha)=\alpha$. Moreover, by changing representatives also for $\zeta_{*}(\beta)=\beta_{1}^{\prime}+\beta_{2}^{\prime}$ as shown in Fig. 7c, e and than doing the above described cut and paste, one can see, in Fig. 7f, that $\beta_{1}^{\prime}+\beta_{2}^{\prime}$ consists of two loops: the first loop included in the left torus and the second in the right torus. The first loop is homologous to the loop $\beta_{1}^{\prime \prime}$ and the second to $\beta_{2}^{\prime \prime}$ in Fig. $7 \mathrm{~g}$, h. It follows that

$$
\zeta_{*}(\beta)=\beta_{1}^{\prime}+\beta_{2}^{\prime}=\beta_{1}^{\prime \prime}+\beta_{2}^{\prime \prime}=\beta-\alpha .
$$


The same conclusion can be obtained also computing the intersection numbers of $\zeta_{*}(\beta)=\beta_{1}^{\prime}+\beta_{2}^{\prime}$ with the generators $\alpha$ and $\beta$ and using Fig. 7f. Indeed,

$$
\left\langle\zeta_{*}(\beta), \alpha\right\rangle=\left\langle\beta_{1}^{\prime}, \alpha\right\rangle+\left\langle\beta_{2}^{\prime}, \alpha\right\rangle=-2 \quad \text { and }\left\langle\zeta_{*}(\beta), \beta\right\rangle=\left\langle\beta_{1}^{\prime}, \beta\right\rangle+\left\langle\beta_{2}^{\prime}, \beta\right\rangle=-2 \text {. }
$$

Since $\zeta_{*}(\beta) \in H_{1}^{(0)}\left(M\left(h^{+} z\right), \mathbb{Z}\right),\{\alpha, \beta\}$ is the basis of $H_{1}^{(0)}\left(M\left(h^{+} z\right), \mathbb{Z}\right)$ with $\langle\alpha, \beta\rangle=$ 2 , the algebraic intersection form is non-degenerated on $H_{1}^{(0)}\left(M\left(h^{+} z\right), \mathbb{Z}\right)$ and $\left\langle\zeta_{*}(\beta), \alpha\right\rangle=-2=\langle\beta, \alpha\rangle=\langle\beta-\alpha, \alpha\rangle$ and $\left\langle\zeta_{*}(\beta), \beta\right\rangle=-2=-\langle\alpha, \beta\rangle=\langle\beta-\alpha, \beta\rangle$, we conclude that $\zeta_{*}(\beta)=\beta-\alpha$. Therefore, $\left(h^{+}\right)_{*}(z)=\left(h^{+}\right)^{-1}$ if $z \notin S$.

In view of (6) and (8), for any $z \in \mathbb{T}_{0}^{2}$

$$
\left(h^{-}\right)_{*}(z)=\left(\vartheta \cdot h^{+} \cdot \vartheta^{-1}\right)_{*}(z)=\vartheta \cdot\left(h^{+}\right)_{*}\left(\vartheta^{-1} z\right) \cdot \vartheta^{-1} .
$$

Since $S$ is symmetric with respect to the involution $\vartheta, z \in S$ if and only if $\vartheta^{-1} z=\vartheta z \in$ $S$. By (6) and (8), it follows that $\left(h^{-}\right)_{*}(z)=h^{-}$if $z \in S$ and $\left(h^{-}\right)_{*}(z)=\left(h^{-}\right)^{-1}$ if $z \notin S$.

In view of Lemma 3.3, for every $z \in \mathbb{T}_{0}^{2}$ and $n \in \mathbb{Z}$ there exists $m_{n}^{ \pm}(z) \in \mathbb{Z}$ such that

$$
\left(\left(h^{ \pm}\right)_{*}^{-n}(z)\right)^{-1}=\left(h^{ \pm}\right)_{*}^{n}\left(\left(h^{ \pm}\right)^{-n} z\right)=\left(h^{ \pm}\right)^{m_{n}^{ \pm}(z)} .
$$

Lemma 3.4. If $z=(x, y) \in \mathbb{T}_{0}^{2}$ with irrational $x$ and $y$ then

(i) $m_{n}^{ \pm}(z) \rightarrow+\infty$ as $n \rightarrow+\infty$;

(ii) the sequences $\left(m_{n}^{ \pm}(z)\right)_{n \geq 1}$ take all natural values.

Proof. We will give the proof for the matrix $h^{+}$. The case of the matrix $h^{-}$is similar. By Lemma 3.3, for every $z^{\prime} \in \mathbb{T}_{0}^{2}$ we have

$$
\left(h^{+}\right)_{*}\left(z^{\prime}\right)=\left(h^{+}\right)^{2 \mathbf{1}_{S}\left(z^{\prime}\right)-1},
$$

where $\mathbf{1}_{S}$ is the indicator function of the subset $S$. In view of (3), it follows that

$$
\begin{aligned}
\left(h^{+}\right)_{*}^{n}\left(\left(h^{+}\right)^{-n} z\right) & =h_{*}^{+}\left(\left(h^{+}\right)^{-1} z\right) \cdot h_{*}^{+}\left(\left(h^{+}\right)^{-2} z\right) \cdots h_{*}^{+}\left(\left(h^{+}\right)^{-n+1} z\right) \cdot h_{*}^{+}\left(\left(h^{+}\right)^{-n} z\right) \\
& =\left(h^{+}\right)^{\sum_{j=1}^{n}\left(2 \mathbf{1}_{S}(x-j y, y)-1\right)} .
\end{aligned}
$$

Therefore

$$
m_{n}^{+}(z)=\sum_{j=1}^{n}\left(2 \mathbf{1}_{S}(x-j y, y)-1\right)=\sum_{j=1}^{n}\left(2 \mathbf{1}_{S_{y}}(x-j y)-1\right),
$$

where $S_{y}:=\left\{x^{\prime} \in \mathbb{T}:\left(x^{\prime}, y\right) \in S\right\}(\mathbb{T}:=[-1 / 2,1 / 2))$. Since $S_{y}$ is an interval of length $1-|y|$, the mean value of the function $2 \mathbf{1}_{S_{y}}(\cdot)-1$ is $1-2|y|>0$ and, by unique ergodicity of the rotation by $y$ on $\mathbb{T}$, we have

$$
\frac{1}{n} \sum_{j=1}^{n}\left(2 \mathbf{1}_{S_{y}}(x-j y)-1\right) \rightarrow 1-2|y|>0 \text { as } n \rightarrow+\infty .
$$

It follows that

$$
m_{n}^{+}(z)=\sum_{j=1}^{n}\left(2 \mathbf{1}_{S_{y}}(x-j y)-1\right) \rightarrow+\infty \text { as } n \rightarrow+\infty .
$$

Since $m_{0}^{+}(z)=0$ and $m_{n+1}^{+}(z)=m_{n}^{+}(z) \pm 1$, the condition (i) implies (ii). 


\section{Irrational $\lambda$}

The aim of this section is to give the proof of the following result on the existence of ergodic directions on $\widetilde{M(0, \lambda)_{\beta}}$ whenever $\lambda \in(0,1 / 2)$ is irrational.

Theorem 4.1. For every irrational $\lambda \in(0,1 / 2)$ the set of ergodic directions on the surface $\widetilde{M(0, \lambda)_{\beta}}$ is uncountable.

This result follows from Theorem 3.1 and the following auxiliary lemma.

Lemma 4.2. Let $\lambda \in(0,1 / 2)$ be an irrational number and let $J \subset(0,1 / 2)$ be a closed interval. Suppose that $z=(x, y)=g^{-1}(0, \lambda)$ for some $g \in S L_{+}(2, \mathbb{Z})$ so that $y>0$. Then there exist natural numbers $a, b, c, d$ such that if $z^{\prime}=\left(x^{\prime}, y^{\prime}\right)=\left(h_{a, b, c, d}\right)^{-1}(x, y)$ with

$$
h_{a, b, c, d}=\left(h^{+}\right)^{a} \cdot h^{-} \cdot h^{+} \cdot\left(h^{-}\right)^{b} \cdot h^{+} \cdot h^{-} \cdot\left(h^{+}\right)^{c} \cdot\left(h^{-}\right)^{d}
$$

then

$$
y^{\prime} \in J \text { and }\left(h_{a, b, c, d}\right)_{*}\left(z^{\prime}\right) \beta=\beta .
$$

Moreover, the number a can be chosen arbitrary large.

Remark 4.3. Note that the irrationality of $\lambda$ implies the irrationality of $y$. If additionally the matrix $g$ has positive entries then $x$ is also irrational.

Proof of Lemma 4.2. Since $y$ is irrational (see the previous Remark), each orbit of the rotation $\mathbb{T} \ni t \mapsto t-y \in \mathbb{T}$ is dense in $\mathbb{T}=[-1 / 2,1 / 2)$, so there exists a natural number $a$ such that $x-a y=-1 / 2+\varepsilon_{1} \bmod 1$ in $\mathbb{T}$, where

$$
0<\varepsilon_{1}<\frac{1}{2} \min (y, 1 / 2-y) .
$$

In addition, by Lemma 3.4, one can assume that the number $a$ is arbitrarily large and $a^{\prime}:=m_{a}^{+}(z)$ is positive. Then

$$
z_{1}=\left(x_{1}, y_{1}\right):=\left(h^{+}\right)^{-a}(x, y)=(x-a y \bmod 1, y \bmod 1)=\left(-1 / 2+\varepsilon_{1}, y\right)
$$

and

$$
\left(h^{+}\right)_{*}^{a}\left(z_{1}\right)=\left(h^{+}\right)^{a^{\prime}} .
$$

Let

$$
\begin{aligned}
z_{2} & =\left(x_{2}, y_{2}\right):=\left(h^{-}\right)^{-1}\left(x_{1}, y_{1}\right)=\left(x_{1} \bmod 1, y_{1}-x_{1} \bmod 1\right) \\
& =\left(-1 / 2+\varepsilon_{1}, y-1 / 2-\varepsilon_{1}\right) .
\end{aligned}
$$

Since $y<1 / 2$, we have $x_{2}+y_{2}=y-1<-1 / 2$, so that $z_{2} \notin S$. Let

$$
\begin{aligned}
z_{3} & =\left(x_{3}, y_{3}\right):=\left(h^{+}\right)^{-1}\left(x_{2}, y_{2}\right)=\left(x_{2}-y_{2} \bmod 1, y_{2} \bmod 1\right) \\
& =\left(2 \varepsilon_{1}-y, y-1 / 2-\varepsilon_{1}\right) .
\end{aligned}
$$

Since $0<\varepsilon<1 / 2$, we have $x_{3}+y_{3}=\varepsilon_{1}-1 / 2 \in(-1 / 2,0)$, so that $z_{3} \in S$. Moreover, as $2 \varepsilon_{1}<y$, we have $x_{3}=2 \varepsilon_{1}-y<0$. Since $x_{3}$ is irrational (see Remark 4.3 for the 
matrix $g \cdot\left(h^{+}\right)^{a} \cdot h^{-} \cdot h^{+}$with has positive entries), by Lemma 3.4, there exists a natural number $b$ such that $b^{\prime}:=m_{b}^{-}\left(z_{3}\right)>a^{\prime}$ and $y_{3}-b x_{3}=1 / 2-\varepsilon_{2} \bmod 1$ in $\mathbb{T}$, where

$$
0<\varepsilon_{2}<\frac{1}{2} \min \left(\left|x_{3}\right|, 1 / 2-\left|x_{3}\right|\right)=\frac{1}{2} \min \left(y-2 \varepsilon_{1}, 1 / 2-y+2 \varepsilon_{1}\right) .
$$

Therefore

$z_{4}=\left(x_{4}, y_{4}\right):=\left(h^{-}\right)^{-b}\left(x_{3}, y_{3}\right)=\left(x_{3} \bmod 1, y_{3}-b x_{3} \bmod 1\right)=\left(2 \varepsilon_{1}-y, 1 / 2-\varepsilon_{2}\right)$ and

$$
\left(h^{-}\right)_{*}^{b}\left(z_{4}\right)=\left(h^{-}\right)^{b^{\prime}}
$$

Let

$$
\begin{aligned}
z_{5} & =\left(x_{5}, y_{5}\right):=\left(h^{+}\right)^{-1}\left(x_{4}, y_{4}\right)=\left(x_{4}-y_{4} \bmod 1, y_{4} \bmod 1\right) \\
& =\left(2 \varepsilon_{1}+1 / 2+\varepsilon_{2}-y, 1 / 2-\varepsilon_{2}\right) .
\end{aligned}
$$

Since $y<1 / 2$ and $\varepsilon_{1}>0$, we have $x_{5}+y_{5}=2 \varepsilon_{1}+1-y>1 / 2$, so that $z_{5} \notin S$. Let

$$
\begin{aligned}
z_{6} & =\left(x_{6}, y_{6}\right):=\left(h^{-}\right)^{-1}\left(x_{5}, y_{5}\right)=\left(x_{5} \bmod 1, y_{5}-x_{5} \bmod 1\right) \\
& =\left(2 \varepsilon_{1}+1 / 2+\varepsilon_{2}-y,-2\left(\varepsilon_{2}+\varepsilon_{1}\right)+y\right) .
\end{aligned}
$$

Since $0<\varepsilon_{2}<1 / 2$, we have $x_{6}+y_{6}=1 / 2-\varepsilon_{2} \in(0,1 / 2)$, so that $z_{6} \in S$. Since $y_{6}$ is irrational (see Remark 4.3), by Lemma 3.4, there exists a natural number $c$ such that $c^{\prime}:=m_{c}^{-}\left(z_{6}\right)=b^{\prime}-a^{\prime}$. Setting

$$
z_{7}=\left(x_{7}, y_{7}\right):=\left(h^{+}\right)^{-c}\left(x_{6}, y_{6}\right)
$$

we have

$$
\left(h^{+}\right)_{*}^{c}\left(z_{7}\right)=\left(h^{+}\right)^{c^{\prime}} .
$$

Since $x_{7}$ is irrational (see Remark 4.3), there exists a natural number $d$ such that $y_{7}-d x_{7}$ $\bmod 1 \in J$. Let $d^{\prime}:=m_{d}^{-}\left(z_{7}\right)$. Setting

$$
z^{\prime}=\left(x^{\prime}, y^{\prime}\right):=\left(h^{-}\right)^{-d}\left(x_{7}, y_{7}\right)=\left(x_{7}, y_{7}-d x_{7} \bmod 1\right),
$$

we obtain $y^{\prime} \in J$ and

Next note that

$$
\left(h^{-}\right)_{*}^{d}\left(z^{\prime}\right)=\left(h^{-}\right)^{d^{\prime}}
$$

$$
\begin{aligned}
& \left(h_{a, b, c, d}\right)_{*}\left(z^{\prime}\right) \\
& =\left(h^{+}\right)_{*}^{a}\left(z_{1}\right) \cdot h_{*}^{-}\left(z_{2}\right) \cdot h_{*}^{+}\left(z_{3}\right) \cdot\left(h^{-}\right)_{*}^{b}\left(z_{4}\right) \cdot h_{*}^{+}\left(z_{5}\right) \cdot h_{*}^{-}\left(z_{6}\right) \cdot\left(h^{+}\right)_{*}^{c}\left(z_{7}\right) \cdot\left(h^{-}\right)_{*}^{d}\left(z^{\prime}\right) .
\end{aligned}
$$

Since $z_{3}, z_{6} \in S$ and $z_{2}, z_{5} \notin S$, by Lemma 3.3,

$$
\left(h^{+}\right)_{*}\left(z_{3}\right)=h^{+}, \quad\left(h^{-}\right)_{*}\left(z_{6}\right)=h^{-}, \quad\left(h^{-}\right)_{*}\left(z_{2}\right)=\left(h^{-}\right)^{-1}, \quad\left(h^{+}\right)_{*}\left(z_{5}\right)=\left(h^{+}\right)^{-1} .
$$

If we combine this with (10)-(13), we obtain

$$
\left(h_{a, b, c, d}\right)_{*}\left(z^{\prime}\right)=\left(h^{+}\right)^{a^{\prime}} \cdot\left(h^{-}\right)^{-1} \cdot h^{+} \cdot\left(h^{-}\right)^{b^{\prime}} \cdot\left(h^{+}\right)^{-1} \cdot h^{-} \cdot\left(h^{+}\right)^{c^{\prime}} \cdot\left(h^{-}\right)^{d^{\prime}} .
$$

In view of (6), it follows that

$$
\begin{aligned}
\left(h_{a, b, c, d}\right)_{*}\left(z^{\prime}\right) & =\left(h^{+}\right)^{a^{\prime}-1} \cdot \omega \cdot\left(h^{-}\right)^{b^{\prime}-1} \cdot \omega^{-1} \cdot\left(h^{+}\right)^{c^{\prime}} \cdot\left(h^{-}\right)^{d^{\prime}} \\
& =\left(h^{+}\right)^{a^{\prime}-1} \cdot\left(h^{+}\right)^{-b^{\prime}+1} \cdot\left(h^{+}\right)^{c^{\prime}} \cdot\left(h^{-}\right)^{d^{\prime}}=\left(h^{-}\right)^{d^{\prime}} .
\end{aligned}
$$

Consequently, $\left(h_{a, b, c, d}\right)_{*}\left(z^{\prime}\right) \beta=\beta$. 
Proof of Theorem 4.1. Let $J=[1 / 6,1 / 3]$. Using Lemma 4.2, we can construct a sequence $\left(z_{n}\right)_{n \geq 0}$ in $\mathbb{T}_{0}^{2}$ such that $z_{0}=(0, \lambda)$ and for every $n \geq 1$ there exist natural numbers $a_{n}, b_{n}, c_{n}, d_{n}$ with $a_{n} \geq 12$ such that

$$
z_{n}=\left(h_{a_{n}, b_{n}, c_{n}, d_{n}}\right)^{-1} z_{n-1} \quad \text { and } \quad\left(h_{a_{n}, b_{n}, c_{n}, d_{n}}\right)_{*}\left(z_{n}\right) \beta=\beta
$$

and $z_{n}=\left(x_{n}, y_{n}\right) \in(-1 / 2,1 / 2) \times(1 / 6,1 / 3)$.

Let us consider the irrational number

$$
\alpha=\left[0 ; a_{1}, 1,1, b_{1}, 1,1, c_{1}, d_{1}, a_{2}, 1,1, b_{2}, 1,1, c_{2}, d_{2}, \ldots\right] .
$$

We will show that the directional flow along the vector $(1, \alpha)$ on $\widetilde{M(0, \lambda})_{\beta}$ is ergodic. In view of the proof of Lemma 4.2, we have a freedom of choice of $d_{n}$ for fixed $a_{n}, b_{n}, c_{n}$. It follows that the set of ergodic directions is uncountable.

Let $\alpha=\left[0 ; e_{1}, e_{2}, \ldots\right]$. In view of (14), we have

$$
\left(\left(h^{+}\right)^{e_{1}} \cdots\left(h^{-}\right)^{e_{8 n}}\right)^{-1} M(0, \lambda)=M\left(z_{n}\right),\left(\left(h^{+}\right)^{e_{1}} \cdots\left(h^{-}\right)^{e_{8 n}}\right)_{*}^{-1}(0, \lambda) \beta=\beta .
$$

Moreover,

$$
y_{n} \in[1 / 6,1 / 3] \text { and } e_{8 n+1}=a_{n+1} \geq 12 \geq \frac{1+\varepsilon}{1 / 2-y_{n}} \text { with } \varepsilon=1 .
$$

Finally Theorem 3.1 applied to the sequence $\left(k_{n}\right)_{n \geq 1}, k_{n}=8 n$ yields the ergodicity of the directional flow along the vector $(1, \alpha)$.

\section{Rational $\lambda$}

The aim of this section is to describe more precisely a subset of ergodic directions on $\widehat{M(0, \lambda)}{ }_{\beta}$ whenever $\lambda \in(0,1 / 2)$ is rational. Such precise description will help us to show that the Hausdorff dimension of the set of ergodic directions is greater than $1 / 2$.

Notation. For every $\lambda=p / 2 q$ with $0<p<q$ relatively prime natural numbers set

$$
B(\lambda):=\left\{\begin{array}{cc}
(3 q-1,1,1,4 q-1,1,1, q) & \text { if } p \text { is odd } \\
(2 q+a, p-1, p+1,2 q+2 a, p-1, p+1, a) & \text { if } p \text { is even }
\end{array}\right.
$$

where $a$ is the unique natural number satisfying $0<a \leq q$ and $a p=-1 \bmod q$.

Theorem 5.1. Suppose that $\lambda=p / 2 q \in(0,1 / 2)$ with $p, q \in \mathbb{Z}$ relatively prime. For every sequence of natural numbers $\left(n_{k}\right)_{k=1}^{\infty}$ if

$$
\alpha=\left[0 ; B(\lambda), n_{1}, B(\lambda), n_{2}, \ldots, B(\lambda), n_{k}, \ldots\right]
$$

then the directional flow along the vector $(1, \alpha)$ on the $\mathbb{Z}$-cover $\widetilde{M(0, \lambda)} \beta$ given by $\beta$ is ergodic. Moreover, the Hausdorff dimension of the set of such ergodic directions is greater than $1 / 2$. 
The proof of the first part of Theorem 5.1 is rather technical and is postponed until the end of the section, where its more general version Theorem 5.6 is proved. The proof is based on two technical Lemmas 5.3 and 5.5 in which we find explicitly some elements of the Veech group of the translation surface $\widetilde{M(z)} \beta$ when both coordinates of $z$ are rational.

The estimate from below of the Hausdorff dimension in Theorem 5.1 follows immediately from the following result, whose proof is fairly standard, but is included for the convenience of the reader.

Proposition 5.2. For every $\bar{a} \in \mathbb{N}^{m}(m \geq 0)$ and for every $B=b \mathbb{N}+c \subset \mathbb{N}(b, c$ are integer with $b>0$ and $c \geq 0$ ) the Hausdorff dimension of the set

$$
\mathcal{E}(\bar{a})=\left\{\left[0 ; \bar{a}, n_{1}, \bar{a}, n_{2}, \bar{a}, n_{3}, \ldots\right]: n_{i} \in B \text { for } i \geq 1\right\}
$$

is greater than $1 / 2$.

Proof. Let $\bar{a}=a_{1} \ldots a_{m}$. It simplifies the argument, and causes no loss of generality, to assume that $m \geq 3$ is odd. For every $l \in \mathbb{N}$ let us consider the map $\psi_{\bar{a}, l}:[0,1] \rightarrow[0,1]$ given by

$$
\psi_{\bar{a}, l}(x)=\left[0 ; a_{1}, a_{2}, \ldots, a_{m}, l+x\right]=\frac{p_{m}(\bar{a})(l+x)+p_{m-1}(\bar{a})}{q_{m}(\bar{a})(l+x)+q_{m-1}(\bar{a})} .
$$

Then

$$
\psi_{\bar{a}, l}([0,1])=\left[\left[0 ; a_{1}, a_{2}, \ldots, a_{m}, l\right],\left[0 ; a_{1}, a_{2}, \ldots, a_{m}, l+1\right]\right]
$$

and for every $x \in[0,1]$ we have

$$
\psi_{\bar{a}, l}^{\prime}(x)=\frac{1}{\left(q_{m}(\bar{a})(l+x)+q_{m-1}(\bar{a})\right)^{2}} \geq \frac{1}{\left(q_{m}(\bar{a})(l+1)+q_{m-1}(\bar{a})\right)^{2}}=: d_{\bar{a}, l}
$$

and

$$
\psi_{\bar{a}, l}^{\prime}(x) \leq \frac{1}{\left(q_{m}(\bar{a}) l+q_{m-1}(\bar{a})\right)^{2}}<1 / 4 .
$$

For every $u \in \mathbb{N}$ let $B_{u}=b\{1, \ldots, u\}+c$. Then

$$
\mathcal{E}(\bar{a}) \supset \mathcal{E}_{u}(\bar{a})=\bigcap_{k \geq 1} \bigcup_{\left(n_{1}, \ldots, n_{k}\right) \in\left(B_{u}\right)^{k}} \psi_{\bar{a}, n_{1}} \circ \psi_{\bar{a}, n_{2}} \circ \ldots \circ \psi_{\bar{a}, n_{k}}[0,1] .
$$

Let

$$
E_{u}:=\left[\left[0 ; a_{1}, a_{2}, \ldots, a_{m}, b\right],\left[0 ; a_{1}, a_{2}, \ldots, a_{m}, b u+c+1\right]\right] .
$$

In view of (16), $\psi_{\bar{a}, l}\left(E_{u}\right) \subset E_{u}$ for every $l \in B_{u}$ and the intervals $\psi_{\bar{a}, l}\left(E_{u}\right), l \in B_{u}$ are pairwise disjoint. In view of Theorem 9.7 in [2], if $s_{u}>0$ is the unique solution of the equation $\sum_{l=1}^{u} d_{\bar{a}, b l+c}^{s}=1$, then $\operatorname{dim}_{H}\left(\mathcal{E}_{u}(\bar{a})\right) \geq s_{u}$. Since $\sum_{l=1}^{\infty} d_{\bar{a}, b l+c}^{1 / 2}=+\infty$, we can choose $u \in \mathbb{N}$ such that $\sum_{l=1}^{u} d_{\bar{a}, b l+c}^{1 / 2}>1$. Thus $s_{u}>1 / 2$, and we get

$$
\operatorname{dim}_{H}(\mathcal{E}(\bar{a})) \geq \operatorname{dim}_{H}\left(\mathcal{E}_{u}(\bar{a})\right) \geq s_{u}>1 / 2,
$$

as desired. 
From now on, we will deal with square tiled translation surfaces $M(z)$ for which $z=(r / 2 q, s / 2 q) \in \mathbb{T}_{0}^{2}$ with $r, s, q \in \mathbb{Z},|r|,|s|<q, s$ is non-zero and coprime with $q$.

Lemma 5.3. Suppose that at least one number $s$ or $r$ is odd. Let a, b be natural numbers such that

$$
0<a, b \leq 2 q \text { and } r+a s=-q \bmod 2 q, \quad b s+s-q=r \bmod 2 q .
$$

Then setting

$$
g_{z}:=\left(h^{+}\right)^{2 q+b} \cdot h^{-} \cdot h^{+} \cdot\left(h^{-}\right)^{2 q+a+b} \cdot h^{+} \cdot h^{-} \cdot\left(h^{+}\right)^{a} \in S L_{+}(2, \mathbb{Z})
$$

we have

$$
g_{z} \cdot M(z)=M(z) \text { and }\left(g_{z}\right)_{*}(z)=i d .
$$

Proof. Without loss of generality we can assume that $s>0$. If $s<0$ then dealing with the point $-z=(-r / 2 q,-s / 2 q) \in \mathbb{T}_{0}^{2}$ we have $g_{-z} \cdot M(-z)=M(-z)$ and $\left(g_{-z}\right)_{*}(-z)=i d$. As $z \in E$, in view of (9), we obtain $g_{-z} \cdot M(z)=M(z)$ and $\left(g_{-z}\right)_{*}(z)=i d$, which is our claim.

Next note that, by (6),

$$
g_{z}=\left(\vartheta \cdot \widetilde{g}_{z} \cdot \vartheta^{-1}\right) \cdot \widetilde{g}_{z}, \text { where } \widetilde{g}_{z}=\left(h^{-}\right)^{2 q+b} \cdot h^{+} \cdot h^{-} \cdot\left(h^{+}\right)^{a} .
$$

This symmetry of $g_{z}$ combined with the fundamental Lemma 3.3 is the heart of the proof. Indeed, using Lemma 3.3 we will prove that

$$
\widetilde{g}_{z} \cdot M(z)=M(\vartheta z), \quad\left(\widetilde{g}_{z}\right)_{*}(z)=\left(h^{-}\right)^{\tilde{b}} \cdot \omega^{-1} \cdot\left(h^{+}\right)^{\tilde{a}}
$$

for some integer $\tilde{a}, \tilde{b}$. In view of (18), (8) and (6), it follows that

$$
\begin{aligned}
\left(\vartheta \cdot \widetilde{g}_{z} \cdot \vartheta^{-1}\right) M(\vartheta z) & =M(z), \\
\left(\vartheta \cdot \widetilde{g}_{z} \cdot \vartheta^{-1}\right)_{*}(\vartheta z) & =\vartheta \cdot\left(h^{-}\right)^{\tilde{b}} \cdot \omega^{-1} \cdot\left(h^{+}\right)^{\tilde{a}} \cdot \vartheta^{-1}=\left(h^{+}\right)^{\tilde{b}} \cdot \omega \cdot\left(h^{-}\right)^{\tilde{a}} .
\end{aligned}
$$

Combining (3) with (18) and (19) and using again (6), we have

$$
\begin{gathered}
\left(\left(\vartheta \cdot \widetilde{g}_{z} \cdot \vartheta^{-1}\right) \cdot \widetilde{g}_{z}\right) \cdot M(z)=M(z), \\
\left(\left(\vartheta \cdot \widetilde{g}_{z} \cdot \vartheta^{-1}\right) \cdot \widetilde{g}_{z}\right)_{*}(z)=\left(h^{+}\right)^{\tilde{b}} \cdot \omega \cdot\left(h^{-}\right)^{\tilde{a}} \cdot\left(h^{-}\right)^{\tilde{b}} \cdot \omega^{-1} \cdot\left(h^{+}\right)^{\tilde{a}} \\
=\left(h^{+}\right)^{\tilde{b}} \cdot \omega \cdot\left(h^{-}\right)^{\tilde{a}+\tilde{b}} \cdot \omega^{-1} \cdot\left(h^{+}\right)^{\tilde{a}}=\left(h^{+}\right)^{\tilde{b}} \cdot\left(h^{+}\right)^{-(\tilde{a}+\tilde{b})} \cdot\left(h^{+}\right)^{\tilde{a}}=i d,
\end{gathered}
$$

which is our claim.

It remains to prove (18). Since $s$ and $q$ are coprime and $s$ or $r$ is odd, there exist $a, b, a^{\prime}, b^{\prime} \in \mathbb{Z}$ with

$$
0<a, b \leq 2 q \text { and } r+a s=2 q a^{\prime}-q, \quad b s+s-q=2 q b^{\prime}+r .
$$

Thus,

$$
\begin{gathered}
\left(h^{+}\right)^{a}(r / 2 q, s / 2 q)=((r+a s) / 2 q, s / 2 q)=\left(a^{\prime}-1 / 2, s / 2 q\right), \\
\left(h^{-}\right)^{2 q}(s / 2 q, r / 2 q)=(s / 2 q,(r+2 q s) / 2 q)=(s / 2 q, r / 2 q+s), \\
\left(h^{-}\right)^{b}(s / 2 q, s / 2 q-1 / 2)=(s / 2 q,(b s+s-q) / 2 q)=\left(s / 2 q, r / 2 q+b^{\prime}\right) .
\end{gathered}
$$


Hence, in view also of Lemma 3.3, there exist $k, l, m \in \mathbb{Z}$ such that

$$
\begin{array}{ll}
\left(h^{+}\right)^{a} M(z)=M(-1 / 2, s / 2 q), & \left(h^{+}\right)_{*}^{q}(z)=\left(h^{+}\right)^{m}, \\
\left(h^{-}\right)^{2 q} M(\vartheta z)=M(\vartheta z), & \left(h^{-}\right)_{*}^{2 q}(\vartheta z)=\left(h^{-}\right)^{k}, \\
\left(h^{-}\right)^{b} M(s / 2 q, s / 2 q-1 / 2)=M(\vartheta z), & \left(h^{-}\right)_{*}^{b}(s / 2 q, s / 2 q-1 / 2)=\left(h^{-}\right)^{l} .
\end{array}
$$

As $0<s<q$, we have $(-1 / 2, s / 2 q) \in S$ and $(-1 / 2, s / 2 q-1 / 2) \notin S$. By Lemma 3.3 and (2), it follows that

$$
\begin{aligned}
& h^{-} M\left(-\frac{1}{2}, \frac{s}{2 q}\right)=M\left(-\frac{1}{2}, \frac{s}{2 q}-\frac{1}{2}\right), \quad\left(h^{-}\right)_{*}\left(-\frac{1}{2}, \frac{s}{2 q}\right)=h^{-} . \\
& h^{+} M\left(-\frac{1}{2}, \frac{s}{2 q}-\frac{1}{2}\right)=M\left(\frac{s}{2 q}, \frac{s}{2 q}-\frac{1}{2}\right), \quad\left(h^{+}\right)_{*}\left(-\frac{1}{2}, \frac{s}{2 q}-\frac{1}{2}\right)=\left(h^{+}\right)^{-1} .
\end{aligned}
$$

Using consequently (21), (24), (25), (23), (22) and (6), we have

$$
\widetilde{g}_{z} \cdot M(z)=\left(\left(h^{-}\right)^{2 q} \cdot\left(h^{-}\right)^{b} \cdot h^{+} \cdot h^{-} \cdot\left(h^{+}\right)^{a}\right) \cdot M(z)=M(\vartheta z)
$$

and

$$
\begin{aligned}
\left(\widetilde{g}_{z}\right)_{*}(z) & =\left(\left(h^{-}\right)^{2 q} \cdot\left(h^{-}\right)^{b} \cdot h^{+} \cdot h^{-} \cdot\left(h^{+}\right)^{a}\right)_{*}(z) \\
& =\left(h^{-}\right)^{k} \cdot\left(h^{-}\right)^{l} \cdot\left(h^{+}\right)^{-1} \cdot h^{-} \cdot\left(h^{+}\right)^{m}=\left(h^{-}\right)^{k+l-1} \cdot \omega^{-1} \cdot\left(h^{+}\right)^{m} .
\end{aligned}
$$

This yields (18), and the proof is complete.

Remark 5.4. Note that if $r=0$ then (20) holds for $a=q$ and $b=q-1$.

Lemma 5.5. Suppose that $r$ and $s$ are both even. Let $a, b$ be natural numbers such that

$$
0<a, b \leq q, \quad b=|s|, \quad \text { and } a s+r=-1 \quad \bmod q .
$$

Then setting

$$
g_{z}:=\left(h^{+}\right)^{2 q+a} \cdot\left(h^{-}\right)^{b-1} \cdot\left(h^{+}\right)^{b+1} \cdot\left(h^{-}\right)^{2 q+2 a} \cdot\left(h^{+}\right)^{b-1} \cdot\left(h^{-}\right)^{b+1} \cdot\left(h^{+}\right)^{a}
$$

we have

$$
g_{z} \cdot M(z)=M(z) \quad \text { and } \quad\left(g_{z}\right)_{*}(z)=i d .
$$

Proof. Without loss of generality we can assume that $s>0$, otherwise we can use again (9) to pass to the positive case. that

The general strategy is much the same as for the proof of Lemma 5.3. Indeed, note

$$
g_{z}=\left(\vartheta \cdot \widetilde{g}_{z} \cdot \vartheta^{-1}\right) \cdot \widetilde{g}_{z}, \quad \text { where } \widetilde{g}_{z}=\left(h^{-}\right)^{2 q+a} \cdot\left(h^{+}\right)^{b-1} \cdot\left(h^{-}\right)^{b+1} \cdot\left(h^{+}\right)^{a}
$$

and we only need to show

$$
\widetilde{g}_{z} \cdot M(z)=M(-\vartheta z), \quad\left(\widetilde{g}_{z}\right)_{*}(z)=\left(h^{-}\right)^{\tilde{b}} \cdot \omega^{-1} \cdot\left(h^{+}\right)^{\tilde{a}}
$$


for some integer $\tilde{a}, \tilde{b}$. Indeed, applying (8) together with (6) we have

$$
\left(\vartheta \cdot \widetilde{g}_{z} \cdot \vartheta^{-1}\right) M(\vartheta z)=M(-z), \quad\left(\vartheta \cdot \widetilde{g}_{z} \cdot \vartheta^{-1}\right)_{*}(\vartheta z)=\left(h^{+}\right)^{\tilde{b}} \cdot \omega \cdot\left(h^{-}\right)^{\tilde{a}}
$$

Since $\vartheta z,-z \in E$, in view of (9), it follows that

$$
\left(\vartheta \cdot \widetilde{g}_{z} \cdot \vartheta^{-1}\right) M(-\vartheta z)=M(z), \quad\left(\vartheta \cdot \widetilde{g}_{z} \cdot \vartheta^{-1}\right)_{*}(-\vartheta z)=\left(h^{+}\right)^{\tilde{b}} \cdot \omega \cdot\left(h^{-}\right)^{\tilde{a}} .
$$

Combining (3) with (27) and (28), and using again (6), we have

$$
\begin{gathered}
\left(\left(\vartheta \cdot \widetilde{g}_{z} \cdot \vartheta^{-1}\right) \cdot \widetilde{g}_{z}\right) \cdot M(z)=M(z), \\
\left(\left(\vartheta \cdot \widetilde{g}_{z} \cdot \vartheta^{-1}\right) \cdot \widetilde{g}_{z}\right)_{*}(z)=\left(h^{+}\right)^{\tilde{b}} \cdot \omega \cdot\left(h^{-}\right)^{\tilde{a}} \cdot\left(h^{-}\right)^{\tilde{b}} \cdot \omega^{-1} \cdot\left(h^{+}\right)^{\tilde{a}}=i d,
\end{gathered}
$$

which is our claim.

It remains to prove (27). As $s$ is even and coprime with $q, q$ is odd. Since $r+1$ is also odd, there exist integers $a, a^{\prime}$ with

$$
a^{\prime} \text { is odd }, \quad 0<a \leq q \text { and } a s+r=a^{\prime} q-1,
$$

and set $b:=s$. Since

$$
\begin{aligned}
\left(h^{+}\right)^{a}(r / 2 q, s / 2 q) & =((r+a s) / 2 q, s / 2 q)=\left(-1 / 2 q+a^{\prime} / 2, s / 2 q\right), \\
\left(h^{-}\right)^{2 q}(-s / 2 q,-r / 2 q) & =(-s / 2 q,-r / 2 q-s), \\
\left(h^{-}\right)^{a}(-s / 2 q, 1 / 2-1 / 2 q) & =(-s / 2 q,(q-1-a s) / 2 q) \\
& =\left(-s / 2 q,\left(1-a^{\prime}\right) / 2-r / 2 q\right),
\end{aligned}
$$

by Lemma 3.3 and (29), there exists $k, l, m \in \mathbb{Z}$ such that

$$
\begin{array}{ll}
\left(h^{+}\right)^{a} M(z)=M(1 / 2-1 / 2 q, s / 2 q), & \left(h^{+}\right)_{*}^{a}(z)=\left(h^{+}\right)^{m} \\
\left(h^{-}\right)^{2 q} M(-\vartheta z)=M(-\vartheta z), & \left(h^{-}\right)_{*}^{2 q}(-\vartheta z)=\left(h^{-}\right)^{k} \\
\left(h^{-}\right)^{a} M\left(-\frac{s}{2 q}, \frac{1}{2}-\frac{1}{2 q}\right)=M(-\vartheta z), & \left(h^{-}\right)_{*}^{a}\left(-\frac{s}{2 q}, \frac{1}{2}-\frac{1}{2 q}\right)=\left(h^{-}\right)^{l} .
\end{array}
$$

One can verify that, for $i, j \in \mathbb{Z}$ with $0 \leq i \leq j<q$, one has

$$
\begin{aligned}
\left(h^{+}\right)^{i}\left(\frac{j}{2 q}, \frac{1}{2}-\frac{1}{2 q}\right) & =\left(\frac{j}{2 q}+\frac{i}{2}-\frac{i}{2 q} \bmod 1, \frac{1}{2}-\frac{1}{2 q} \bmod 1\right) \\
& = \begin{cases}\left(\frac{j-i}{2 q}, \frac{1}{2}-\frac{1}{2 q}\right), & \text { for } i \text { even, } \\
\left(\frac{j-i}{2 q}-\frac{1}{2}, \frac{1}{2}-\frac{1}{2 q}\right), & \text { for } i \text { odd. }\end{cases}
\end{aligned}
$$

If in addition $i<j$, one can also check that

$$
\left(\frac{j-i}{2 q}, \frac{1}{2}-\frac{1}{2 q}\right) \notin S, \quad\left(\frac{j-i}{2 q}-\frac{1}{2}, \frac{1}{2}-\frac{1}{2 q}\right) \in S .
$$

Thus, by Lemma 3.3, we have

$$
\left(h^{+}\right)_{*}\left(\left(h^{+}\right)^{i}\left(\frac{j}{2 q}, \frac{1}{2}-\frac{1}{2 q}\right)\right)= \begin{cases}\left(h^{+}\right)^{-1} & \text { for } i \text { even, } \\ h^{+} & \text {for } i \text { odd. }\end{cases}
$$


Since $1 \leq b=s<q$ and $s$ is even by assumption, it follows by induction that

$$
\begin{aligned}
\left(h^{+}\right)^{b} M\left(\frac{s}{2 q}, \frac{1}{2}-\frac{1}{2 q}\right) & =M\left(0, \frac{1}{2}-\frac{1}{2 q}\right), \\
\left(h^{+}\right)_{*}^{b}\left(\frac{s}{2 q}, \frac{1}{2}-\frac{1}{2 q}\right) & =i d ; \\
\left(h^{+}\right)^{b-1} M\left(\frac{1}{2}-\frac{1}{2 q}, \frac{1}{2}-\frac{1}{2 q}\right) & =M\left(-\frac{s}{2 q}, \frac{1}{2}-\frac{1}{2 q}\right), \\
\left(h^{+}\right)_{*}^{b-1}\left(\frac{1}{2}-\frac{1}{2 q}, \frac{1}{2}-\frac{1}{2 q}\right) & =\left(h^{+}\right)^{-1} .
\end{aligned}
$$

Thus, recalling (6) and applying (8) to (33), we have

$$
\left(h^{-}\right)^{b} M\left(\frac{1}{2}-\frac{1}{2 q}, \frac{s}{2 q}\right)=M\left(\frac{1}{2}-\frac{1}{2 q}, 0\right), \quad\left(h^{-}\right)_{*}^{b}\left(\frac{1}{2}-\frac{1}{2 q}, \frac{s}{2 q}\right)=i d .
$$

In view of Lemma 3.3, we have also

$$
h^{-} M\left(\frac{1}{2}-\frac{1}{2 q}, 0\right)=M\left(\frac{1}{2}-\frac{1}{2 q}, \frac{1}{2}-\frac{1}{2 q}\right), \quad\left(h^{-}\right)_{*}\left(\frac{1}{2}-\frac{1}{2 q}, 0\right)=h^{-} .
$$

Thus, using consequently (30), (35), (36), (34), (32) and (31), we have

$$
\left(\widetilde{g}_{z}\right) \cdot M(z)=\left(\left(h^{-}\right)^{2 q+a} \cdot\left(h^{+}\right)^{b-1} \cdot h^{-} \cdot\left(h^{-}\right)^{b} \cdot\left(h^{+}\right)^{a}\right) \cdot M(z)=M(-\vartheta z)
$$

and using the same series of equations together with (6) we also have

$$
\begin{aligned}
\left(\widetilde{g}_{z}\right)_{*}(z) & =\left(\left(h^{-}\right)^{2 q+a} \cdot\left(h^{+}\right)^{b-1} \cdot h^{-} \cdot\left(h^{-}\right)^{b} \cdot\left(h^{+}\right)^{a}\right)_{*}(z) \\
& =\left(h^{-}\right)^{k+l} \cdot\left(h^{+}\right)^{-1} \cdot h^{-} \cdot i d \cdot\left(h^{+}\right)^{m}=\left(h^{-}\right)^{k+l-1} \cdot \omega^{-1} \cdot\left(h^{+}\right)^{m} .
\end{aligned}
$$

This yields (27) and completes the proof.

Notation. Let $z=(r / 2 q, s / 2 q) \in \mathbb{T}_{0}^{2}$, where $r, s, q$ are integer numbers with $|r|,|s|<$ $q$ and such that $s \neq 0$ is coprime with $q$. Suppose that

$$
B(z):=\left\{\begin{array}{cc}
(2 q+b, 1,1,2 q+a+b, 1,1, a) & \text { if } s \text { or } r \text { is odd } \\
(2 q+a, b-1, b+1,2 q+2 a, b-1, b+1, a) & \text { if } s \text { and } r \text { are even, }
\end{array}\right.
$$

where $a, b$ are the natural numbers satisfying (17) or (26) respectively.

The following result is a more general version of Theorem 5.1.

Theorem 5.6. Suppose that $z=(r / 2 q, s / 2 q) \in \mathbb{T}_{0}^{2}$, where $r, s, q$ are integer numbers with $|r|,|s|<q$. Additionally, assume that $s \neq 0$ is coprime with $q$. For every sequence of natural numbers $\left(n_{k}\right)_{k=1}^{\infty}$ in $2 q \mathbb{N}$ if

$$
\alpha=\left[0 ; B(z), n_{1}, B(z), n_{2}, \ldots, B(z), n_{k}, \ldots\right]
$$

then the directional flow along the vector $(1, \alpha)$ on the $\mathbb{Z}$-cover $\widetilde{M(z)}$ given by $\beta$ is ergodic. Moreover, the Hausdorff dimension of the set of such ergodic directions is greater than $1 / 2$.

Moreover, if $r=0$ then ergodicity holds also for each sequence of natural numbers $\left(n_{k}\right)_{k=1}^{\infty}$.

Proof. By Lemma 3.3,

$$
\left(h^{-}\right)^{n} \cdot M(z)=M(z) \text { and }\left(h^{-}\right)_{*}^{n}(z) \beta=\beta
$$


for every $n \in 2 q \mathbb{N}$. Moreover, if $r=0$ then (38) is valid for every natural $n$. In view of Lemmas 5.3 and 5.5, it follows that

$$
\left(g_{z} \cdot\left(h^{-}\right)^{n}\right) \cdot M(z)=M(z) \quad \text { and } \quad\left(g_{z} \cdot\left(h^{-}\right)^{n}\right)_{*}(z) \beta=\beta .
$$

Setting $\alpha=\left[0, a_{1}, a_{2}, a_{3}, \ldots\right]$, it follows that

$$
\begin{gathered}
\left(\left(h^{+}\right)^{a_{1}} \cdots\left(h^{-}\right)^{a_{8 k}}\right)^{-1} \cdot M(z)=M(z) \\
\left(\left(h^{+}\right)^{a_{1}} \cdots\left(h^{-}\right)^{a_{8 k}}\right)_{*}^{-1}(z) \beta=\beta .
\end{gathered}
$$

for every natural $k$. Moreover,

$$
a_{8 k+1} \geq 2 q+1 \geq \frac{2 q+1}{q-s}=\frac{1+\varepsilon}{\frac{1}{2}-\frac{s}{2 q}} \quad \text { with } \varepsilon=\frac{1}{2 q} .
$$

In view of Theorem 3.1, this gives the ergodicity of the directional flow in the direction of $(1, \alpha)$ on $\widetilde{M(z)} \beta$. The lower bound on the Hausdorff dimension of the set of ergodic directions then follows directly from Proposition 5.2.

Acknowledgements. We would like to thank Pascal Hubert and Barak Weiss who gave us preliminary versions of the paper [9] and explained to us Theorem 2.1, and Vincent Delecroix for useful discussions. This collaboration was supported by the EPSRC Grant EP/I019030/1 and by the Narodowe Centrum Nauki Grant DEC-2011/03/B/ST1/00407.

Open Access This article is distributed under the terms of the Creative Commons Attribution License which permits any use, distribution, and reproduction in any medium, provided the original author(s) and the source are credited.

\section{References}

1. Bachurin, P., Khanin, K., Marklof, J., Plakhov, A.: Perfect retroreflectors and billiard dynamics. J. Mod. Dyn. 5, 33-48 (2011)

2. Falconer, K.: Fractal Geometry. Mathematical Foundations and Applications. John Wiley \& Sons, Inc., Hoboken (2003)

3. Frączek, K., Ulcigrai, C.: Non-ergodic $\mathbb{Z}$-periodic billiards and infinite translation surfaces. Invent. Math. (2013). doi:10.1007/s00222-013-0482-z

4. Fulton, W.: Algebraic Topology. A First Course. Graduate Texts in Mathematics, vol. 153. SpringerVerlag, New York (1995)

5. Gutkin, E.: Geometry, topology and dynamics of geodesic flows on noncompact polygonal surfaces. Regul. Chaotic Dyn. 15, 482-503 (2010)

6. Gutkin, E.: Billiard dynamics: an updated survey with the emphasis on open problems. Chaos 22, 026116 (2012)

7. Hooper, P.: The invariant measures of some infinite interval exchange maps. arXiv:1005.1902

8. Hooper, P., Weiss, B.: Generalized staircases: recurrence and symmetry. Ann. Inst. Fourier (Grenoble) 62, $1581-1600$ (2012)

9. Hubert, P., Weiss, B.: Ergodicity for infinite periodic translation surfaces. Compos. Math. 149, 1364-1380 (2013)

10. Katok, A., Zemljakov, A.: Topological transitivity of billiards in polygons. (Russian) Mat. Zametki 18, 291-300 (1975)

11. Masur, H.: Hausdorff dimension of the set of nonergodic foliations of a quadratic differential. Duke Math. J. 66, 387-442 (1992)

12. Viana, M.: Dynamics of interval exchange transformations and Teichmüller flows. Lecture notes available from http://w3.impa.br/ viana/out/ietf.pdf

13. Yoccoz, J.-C.: Continued Fraction Algorithms for Interval Exchange Maps: An Introduction, Frontiers in Number Theory, Physics, and Geometry. I, pp. 401-435. Springer, Berlin (2006)

14. Yoccoz, J.-C.: Interval exchange maps and translation surfaces. Homogeneous flows, moduli spaces and arithmetic. Clay Math. Proc. 10, 1-69 (2010)

15. Zorich, A.: Flat Surfaces, Frontiers in Number Theory, Physics, and Geometry. I, pp. 437-583. Springer, Berlin (2006) 